\title{
A novel contact algorithm based on a distance potential function for the 3D discrete element method
}

\author{
Lanhao Zhao ${ }^{\mathrm{a}}$, Xunnan Liu ${ }^{\mathrm{a}}$, Jia Mao ${ }^{\mathrm{a}}$, Dong Xü ${ }^{\mathrm{b}}$, Antonio. Munjiza ${ }^{\mathrm{c}}$, Eldad. Avital ${ }^{\mathrm{d}}$ \\ ${ }^{\text {a }}$ College of Water Conservancy and Hydropower, Hohai University, Nanjing, 210098, China \\ ${ }^{\mathrm{b}}$ State Key Laboratory of Hydraulic Engineering Simulation and Safety, Tianjin University, Tianjin, 300072, China \\ ${ }^{\mathrm{c}}$ Faculty of Civil Engineering, University of Split, Croatia \\ ${ }^{\mathrm{d}}$ School of Engineering and Materials Science, Queen Mary University of London, London, E1 4NS, United Kingdom
}

\begin{abstract}
The combined finite-discrete element method (FDEM) has made a groundbreaking progress in the computation of contact interaction. However, FDEM has a strict requirement on the element type, and the simulation result may be inconsistent due to a deficiency of physical meaning of the potential function. To address this problem, a new 3D discrete element method based on a distance potential is proposed for a system consisting of a large number of arbitrary convex polyhedral elements. In this approach, a welldefined distance potential is proposed as a function of the penetration between the contact couples. It exhibits a clear physical meaning and precise measurement of the embedding between the elements in contact. The newly presented method provides a holonomic and accurate contact interaction without being influenced by the element shape. Therefore, the restraint of the element type in FDEM is removed and the proposed method can be used for arbitrary convex polyhedrons. In addition, an improved contact detection algorithm for non-uniform block discrete elements is provided to overcome the demand of the elements with same size of the Munjiza-No Binary Search (Munjiza-NBS) contact detection method. The new approach retains the merits of the FDEM and avoids its deficiencies. It is validated with wellknown benchmark examples including an impact simulation, a friction experiment, a joints structure of a sliding rock mass, pillar impact, block accumulation and analysis for the failure process of wedge slope. The results of this proposed method are in excellent agreement with existing experimental measurements and analytical solutions.
\end{abstract}

Keywords: discrete-element modelling; arbitrary convex polyhedral element; distance potential function; tangential contact interaction; contact detection algorithm

* Corresponding author:

Lanhao Zhao

Tel.: +86 13914733646

Fax: +862583786281

E-mail address: zhaolanhao@hhu.edu.cn 


\section{Introduction}

Computational discontinuous mechanics has an important role in various engineering fields. Generally, the analyzed medium is discretized into a large number of individual blocks using discontinuous structural planes. The mechanical behavior of separate entities is hard to model with traditional numerical methods based on the continuum assumption. By comparison, discontinuous methods apply the block or particle-based models to describe the movement of each component. Over recent decades, development of accurate and efficient numerical methods for discontinuous problems has become a very active research area of computational mechanics.

In general, discontinuous numerical methods can be classified as implicit or explicit methods according to the solution methods for dynamic equations. Among the implicit approaches, the discontinuous deformation analysis (DDA) originated by Shi (Shi and Goodman 1985; Shi 2001) is a typical method. Since the initiation of the two dimensional model for DDA in 1985, diverse applications have been carried out to validate the performance for 2D problems (Zhang et al. 2014; Nie et al. 2014; Morgan and Aral 2015). Although much attention has been paid (Yeung et al. 2003; Yeung et al. 2007; Zhu et al. 2016), DDA still suffers from many deficiencies when it is extended to three dimensions due to a serious obstacle in the contact detection. In DDA, as the contact condition is determined by the process of open-close iteration (Zheng et al. 2016), the stiffness of the contact spring significantly affects the results. The open-close iteration is not always convergent, especially when many point-to-point contact candidates are involved in the problem. Many improvements have been applied to avoid the contact spring (Lin et al. 1996; Cai et al. 2000; Zheng and Jiang 2009), but the open-close iteration is still necessary to determine the contact state and it may fail due to rank deficiency of the stiffness matrix (Zheng et al. 2016; Li and Zheng 2015; Zheng and Li 2015). Furthermore, it has no efficient way to solve the point-to-point contact condition (Bao and Zhao 2012). Originally, the stress and strain inside the block are assumed to be constant and the deformation of blocks are limited in DDA due to the utilization of a first-order displacement function. To overcome these drawbacks, Shi (Shi 1992) proposed the numerical manifold method (NMM) based on the finite cover approximation theory. The 2D NMM has already been developed and used in various researches (Terada et al. 2003; Ning et al. 2011; Zheng and $\mathrm{Xu}$ 2014). On the contrary, the three dimensional NMM is in early development stage, and the published literature is still focused on the concept extension and equation derivation (Jiang et al. 2009; He et al. 2013).

An alternative to the discontinuous numerical method is the discrete element method (DEM). Instead of the implicit approach utilized in DDA and NMM, an explicit scheme is employed to avoid the establishment and resolution of a motion matrix equation (Jing 2003). The non-linearity in material behavior can also be handled in a straightforward manner. Depending upon the description of the geometrical configuration in elements, DEM is generally divided into two categories, namely granular discrete method and block discrete element method. The granular discrete element method, firstly suggested by Cundall and Strack (Cundall and Strack 1979), has enjoyed considerable popularity due to the simplicity of the element type (Smeets et al. 2015) and efficiency in contact detection (Hohner et al. 2011). The commercial code, Particle flow code PFC2D/3D (Itasca 2014a; Itasca 2014b), developed by Itasca Consulting Group, has been widely applied to simulate the motion of rock-mass (Albusaidi et al. 
2005; Shimizu et al. 2011; Yoon et al. 2014) and soil materials (Mcdowell and Harireche 2002; Jung et al. 2012; Wang and Yan 2013). Subsequently, multi-element particle models were also developed (Garcia et al. 2009; Zhou et al. 2013). However, the granular discrete element method has a severe drawback that its spherical element is not able to capture the complex physical features of an actual particle, such as interlocking of particles (Latham and Munjiza 2004). In addition, the value of shear strength among the granular particle assemblies are below those obtained experimentally (Jiang et al. 2015).

On the other hand, the block discrete element method raised by Cundall and Strack (Cundall 1971; Cundall 1988; Hart et al. 1988) has received a close attention because it considers realistic element shapes for a more accurate interaction. The core concept of the method is that it treats all blocks as rigid and a small amount of overlap is allowed in order to consider relative displacement. At each time step, the contact interaction is determined by laws which update the contact forces based on the relative motions and relevant contact stiffness. This method has already been applied in the commercial codes UDEC (Cundall and Hart 1985; Itasca 2016a) and 3DEC (Itasca 2016b), and it has a broad variety of applications in engineering and research studies (Zhu et al. 2013; Boon et al. 2014).

However, the standard DEM lacks a simple and unified mathematical model of the contact interaction in different contact statuses. Normal and tangential springs are used to express the contact force. As a result, different calculation models, such as point-to-point, point-to-edge, point-to-face, are not robust and generally very complex, but are necessary according to the different contact situations. Furthermore, it cannot deal with the point-to-point contact state. For the standard block DEM method, this kind of difficulty mainly comes from the non-determinacy of the normal contact force, as the normal direction does not vary smoothly but discontinuously at a corner. This also results in an inconsistent contact force which can cause energy imbalance and numerical errors. One common practice to overcome the corner singularity is to employ a corner rounding procedure so that blocks can slide past one another in a smooth way when two opposing corners interact (Bao and Zhao 2012). However, this practice has a negative effect on the accuracy and robustness. In order to address complex contact modes, Cundall (Cundall 1988) introduced the common plane (CP) method. By translating and rotating the common plane, the contact types can be reduced to a corner-to-plane contact. However, the common plane method and the improved algorithms still face a number of problems (Nezami et al. 2004; Nezami et al. 2006), for example, the proper choice of the common plane, the iterative error for the spinning common plane and its uniqueness. Consequently, the reliability of simulation results would be influenced by these defects.

Some improvements have been introduced to make DEM more accordant with practical circumstances and provide more efficient models both in contact interaction (Li et al. 2004; Jin et al. 2011; Feng et al. 2012; Lu et al. 2015; Kawamoto et al. 2016) and contact detection (Kodam et al. 2010a; Kodam et al. 2010b). Among them, the combined finite-discrete element method (FDEM) draws the most attention after it was introduced by Munjiza (Munjiza et al. 1995). Entirely different from the standard DEM, FDEM defines a new potential function instead of employing normal or tangential spring. Accordingly, the contact force in FDEM is determined conveniently and uniformly by an integral of the potential function over the embedded volume. In addition, each element is discretized into finite elements to capture the deformability of an individual discrete element. Generally, Munjiza (Munjiza et al. 1995) 
has made a revolutionary change on the mode of contact interaction which establishes a new and uniform calculation model to avoid the discussion of different contact situations completely, whilst the energy conservation and momentum balance properties are preserved during the contact process. In the meantime, to improve the efficiency of contact detection, Munjiza (Munjiza and Andrews 1998) produced a linear algorithm, which is known as No Binary Search (NBS) (Munjiza and Andrews 1998). This new algorithm performs well both in dense and loose packs of blocks because of the insensitivity of the spatial distribution of blocks (Munjiza et al. 2006) and it can reduce the CPU requirement of contact problems (Munjiza 2004). The research code Y-code has been developed by Munjiza in 2004 (Munjiza 2004), together with a graphical user interface (Y-GUI) which is a practical example of this approach (Mahabadi et al. 2010).

Although various applications have been published to represent the validations of FDEM (Munjiza and John 2002; Munjiza et al. 2004; Rougier et al. 2011; Mahabadi et al. 2012), the numerical precision of this method remains uncertain. As the potential function is defined in a simple format which is the normalized penetrated volume, the potential value is greatly influenced by the element shape. In other words, with the same penetration and overlapping volume within a same element, the potential magnitude is not identical at all time. Therefore, both the contact interaction and the simulation may undergo deviations which can decrease computational accuracy, even though the macroscopic mechanical behaviors of blocks is achieved conveniently and quickly in most cases. The principal reason for this phenomenon fundamentally boils down to the inherent deficiency of the potential definition, which lacks a clear physical meaning and a measurement for the penetration between the contact couples. Besides, this numerical model of contact interaction is incomplete, because this method does not indicate the influence of tangential contact force. Another severe drawback is that FDEM cannot be used for an arbitrary polyhedral element due to the specific definition of the potential function for the four-node tetrahedron (Munjiza 2004). Moreover, the NBS contact detection algorithm is not efficient when it is utilized to deal with a system of a large number of polyhedral blocks with different sizes. It limits the application of this method in engineering due to the rapid increase of computing time.

In the current work, a three-dimensional distance potential discrete element method has been proposed. In this approach, a novel definition of distance potential function is developed and a complete calculation algorithm for the normal contact interaction is exhibited. Furthermore, the proposed method also provides a precise definition of the tangential direction and the computational algorithm. The new approach retains the merit of FDEM and avoids its deficiencies. The new function is based on the normalized penetrated distance. In comparison with the definition in FDEM, this function exhibits a clear physical meaning and presents an accurate measurement of penetration for the contact elements. Accordingly, both the magnitude of the distance potential function and numerical solution of the contact interaction are calculated regardless of the element types and shapes. Quite apart from that, a non-uniform block contact detection algorithm is introduced to overcome the defects of NBS contact detection algorithm.

This paper is organized as follows. The basic idea of FDEM is given in section 2 in which the definition of the potential function and its calculation algorithm are introduced. Then the new distance potential function and the algorithm of contact interactions are derived in detail in section 3 . An improved 
new non-uniform blocks search algorithm is introduced in section 4. Several illustrative examples are applied to validate the presented method in section 5. Brief conclusions are summarized in section 6 .

\section{Basic idea of FDEM}

\subsection{Definition of potential function and contact force calculation}

The most notable difference between FDEM and the classic discrete element method lies in the application of a potential function. Instead of the force-displacement law, FDEM employs the definition of a potential function and a potential contact force to calculate the contact interaction.

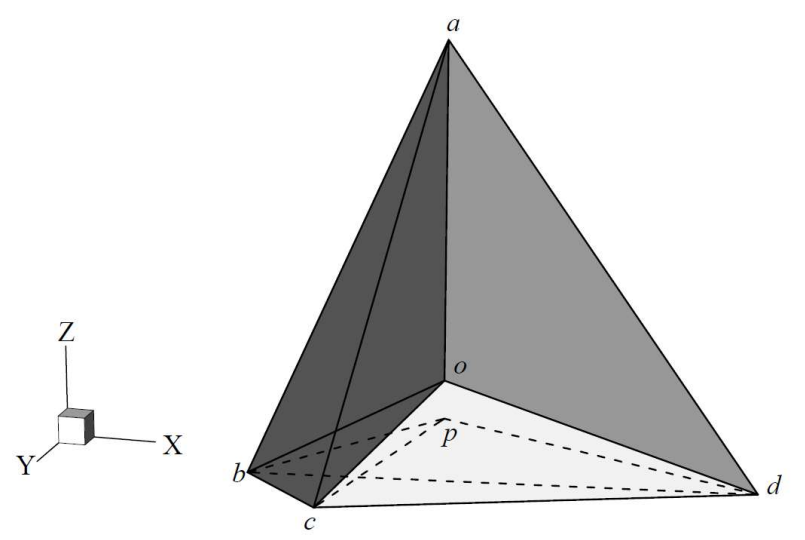

Fig. 1 Sub-tetrahedral elements divided by a corresponding centroid $o$ and a point $p$ in sub-tetrahedron

$$
o-b-c-d
$$

As shown in Fig. 1, a tetrahedral element is separated into four-node sub-tetrahedral elements according to the corresponding centroid $o$. The potential value of each point $p$ in the sub-tetrahedron is defined as:

$$
\varphi(p)=k\left(\frac{V_{p-b-c-d}}{4 V_{o-b-c-d}}\right)
$$

where $k$ is the penalty parameter, $V_{p-b-c-d}$ and $V_{o-b-c-d}$ stand for the volume of tetrahedron $p-b-c-d$ and sub-tetrahedron $o-b-c-d$, respectively.

Consider two discrete elements, $\beta_{t}$ and $\beta_{c} . \beta_{t}$ is penetrated by the element $\beta_{c}$, as shown in Fig. 2. 


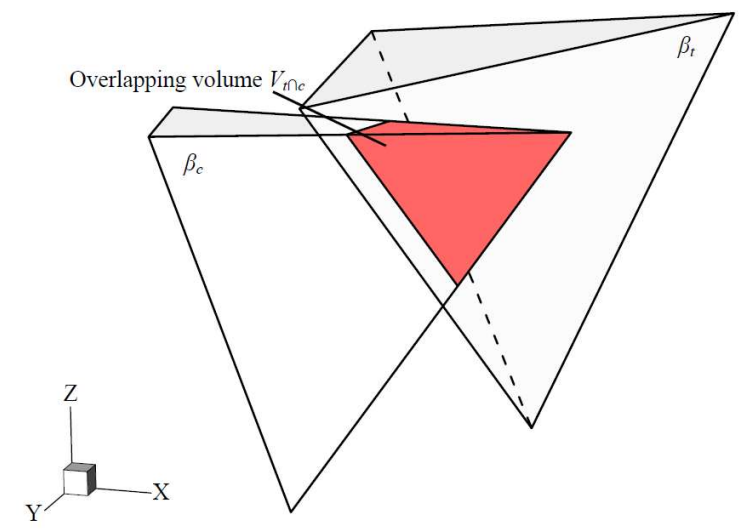

Fig. 2 The contact couple and their overlapping volume.

The normal contact force acting on the overlapping domain is generated by integrating $\varphi$ over the overlapping volume $V_{t \cap c}$, as follows:

$$
\boldsymbol{f}_{n}=\int_{V_{t \cap c}}\left[\operatorname{grad} \varphi_{c}-\operatorname{grad} \varphi_{t}\right] d V,
$$

where $\varphi_{c}$ and $\varphi_{t}$ are the corresponding potential functions in $V_{t \cap c}$ belonging to the elements $\beta_{c}$ and $\beta_{t}$, respectively.

Eq.(2) can also be rewritten as an integration over the boundary surface $S_{t \cap c}$ of $V_{t \cap c}$ in accordance with the Gauss formula:

$$
\boldsymbol{f}_{n}=\int_{S_{t \cap c}} \boldsymbol{n}\left(\varphi_{c}-\varphi_{t}\right) d S
$$

where $\boldsymbol{n}$ is the outward unit vector of the boundary surface $S_{t \cap c}$.

\subsection{Some issues of contact force in FDEM}

In the context of FDEM, the potential ${ }^{\varphi}$ plays an important role in calculating the normal contact force. From Eq.(1), it is clear that $\varphi$ is a function of the penetrated distance and itself provides an extent measurement of the embeddedness between the contact couples. It means that the potential gradient should be constant and the points with the same penetrated distance ought to have the same magnitude within the same element. The potential distributions in tetrahedral elements are exhibited in Fig. 3. It is worthwhile to notice that the distribution density of potential remains constant inside the regular tetrahedral element in Fig. 3(a), however, high difference is indicated in every sub-tetrahedron of the arbitrary tetrahedral element in Fig. 3(b). Accordingly, this approach is strongly sensitive to the element's shape. 


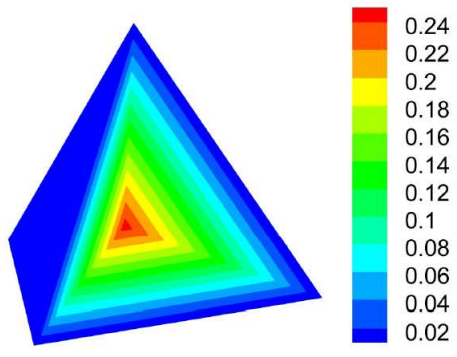

(a)

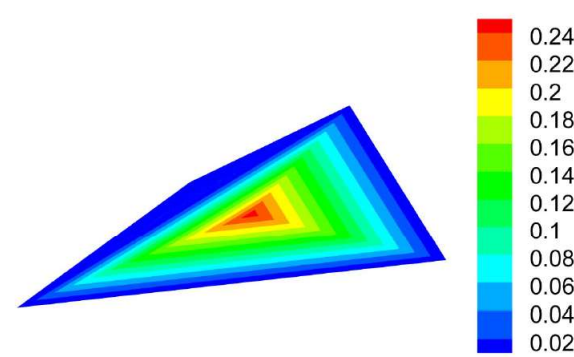

(b)

Fig. 3 The potential distribution in tetrahedral elements. (a) potential distribution in a regular tetrahedron; (b) potential distribution in an arbitrary tetrahedron.

The reason for this phenomenon lies in the inherent defect of this potential definition. As shown in Fig.

4

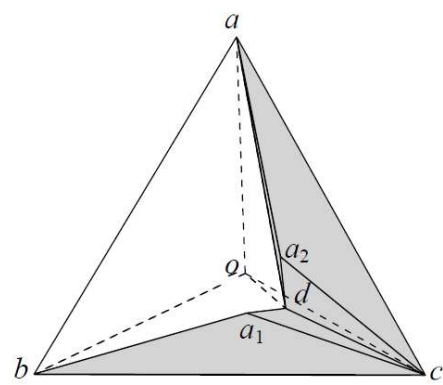

(a)

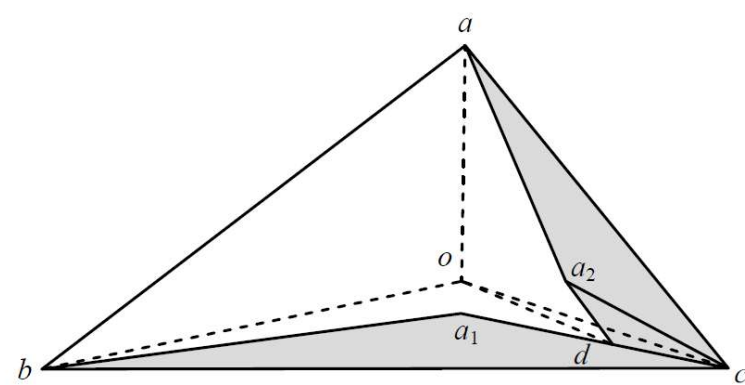

(b)

Fig. 4, points $a_{1}$ and $a_{2}$ are selected with the same distance $h$ along the inward unit normal vector of the bases $b-c-d, a-c-d$ in tehrahedons $a-b-c-d$, respectively. The potential value at points $a_{1}$ and $a_{2}$ can be calculated using Eq.(1):

$$
\varphi\left(a_{1}\right)=k\left(\frac{V_{a_{1}-b-c-d}}{4 V_{o-b-c-d}}\right), \varphi\left(a_{2}\right)=k\left(\frac{V_{a_{2}-a-c-d}}{4 V_{o-a-c-d}}\right) .
$$

The different shapes of the tetrahedron results in different results. On the basis of the geometrical property of tetrahedron, volumes of tetrahedra $a_{1}-b-c-d$ and $a_{2}-a-c-d$ shown in Fig. 4(a) are equivalent, but they are different in Fig. 4(b). Consequently, in Fig. 4(a), $\varphi\left(a_{1}\right)=\varphi\left(a_{2}\right)$, but they are not equal in Fig. 4(b). The discrepancy caused by the element shape can be observed clearly. As a result, the normal contact interactions are also not equivalent by Eq.(3). In fact, although the element shapes are different, the normal contact force cannot be different because of the same penetrated distance. 


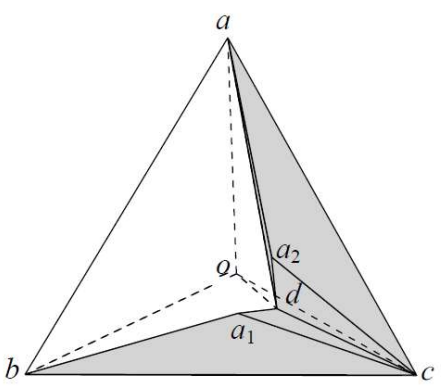

(a)

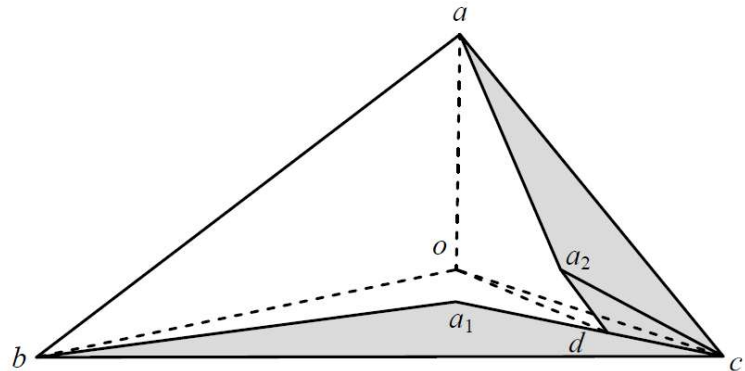

(b)

Fig. 4 Points $a_{1}$ and $a_{2}$ with a same penetrated distance $h$ in tetrahedron $a-b-c-d$. (a) $a-b-c-d$ is a regular tetrahedron; (b) $a-b-c-d$ is an arbitrary tetrahedron.

In addition, this potential definition has a strict restriction to the element type and cannot be used to compute the contact interaction among polyhedra. The fundamental cause for this problem comes from the basic concept in Eq.(1). As shown in Fig. 5, the hexahedron is discretized into five-node pyramidal blocks. Analogously, two points $p_{1}$ and $p_{2}$ with same penetrated distance are selected. The potential value for $p_{1}$ and $p_{2}$ can be obtained by Eq.(1).
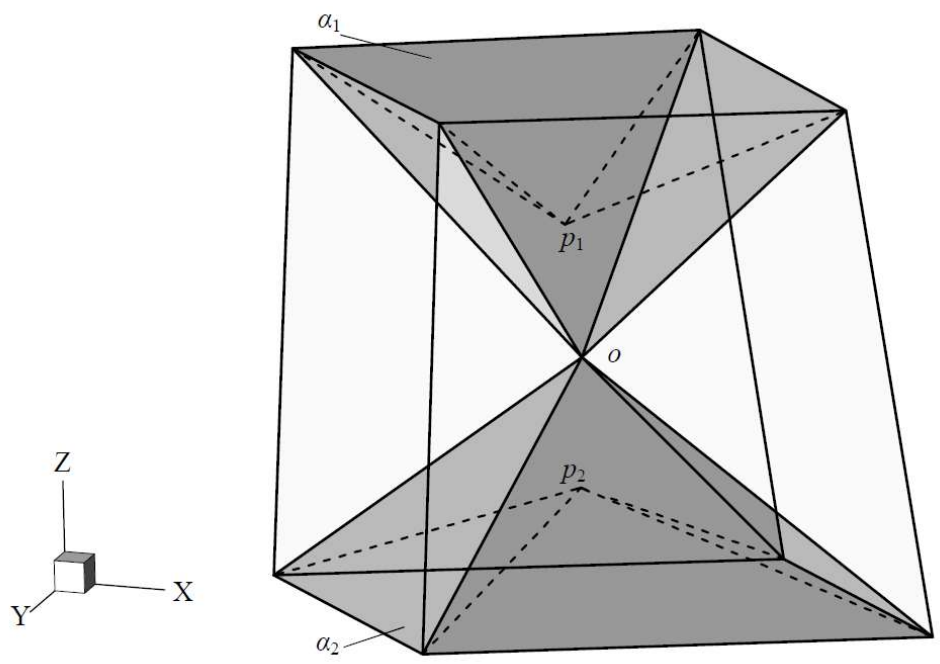

Fig. 5 Sub-polyhedral blocks divided by the corresponding centroid $o$ and two points $p_{1}$ and $p_{2}$ with a same penetrated distance.

Noted that the same result for points $p_{1}$ and $p_{2}$ cannot be satisfied with Eq.(1) due to the randomicity of boundary surfaces $\alpha_{1}$ and $\alpha_{2}$. More importantly, the presence of discontinuities along the interfaces can be observed in the arbitrary polyhedral block exhibited in Fig. 6(b). 


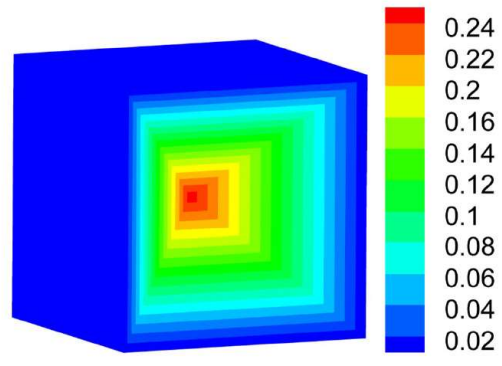

(a)

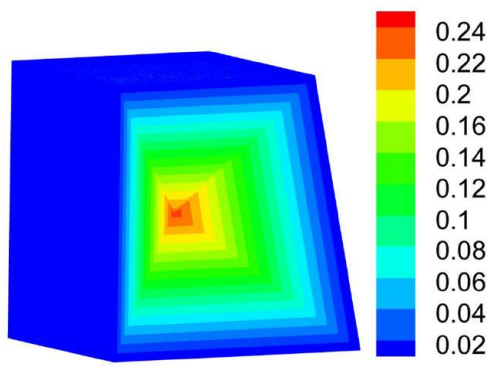

(b)

Fig. 6 The potential distribution in hexahedral elements. (a) potential distribution in a cube; (b) potential distribution in an arbitrary hexahedron.

\section{Distance potential discrete element method}

\subsection{Definition of distance potential function}

As mentioned before, FDEM is an excellent convenient and efficient algorithm for the calculation of contact interaction, however, this method has a strict requirement on the element type and the simulation result may be inconsistent. In this section, a new definition of the distance potential function is developed to overcome these defects.

Assuming an arbitrary convex polyhedral element, the radius of a maximum inscribed spherical element expressed as $r$ is shown in Fig. 7. As described in Fig. 8, the inner domain is surrounded by boundaries which have the same distance $r$ from the base surfaces of the block. The block is discretized into sub-polyhedral elements by the nodes of the bases and inner domain as shown in Fig. 8(b). Accordingly, the distance potential value $\varphi_{d}$ for each point $\mathrm{p}$ in the sub-polyhedron is defined as:

$$
\varphi_{d}(p)=\frac{1}{r} \cdot h_{\mathrm{I}}, \varphi_{d} \leq 1
$$

where $h_{\mathrm{I}}$ is the distance from the point $p$ to the base $\alpha_{\mathrm{I}}$ of the sub-polyhedron.

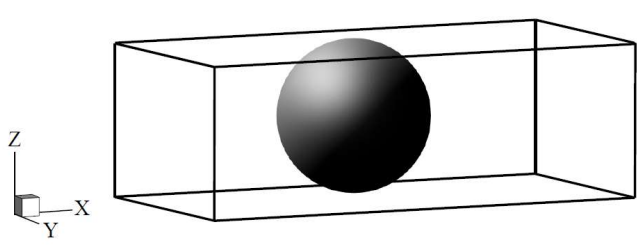

(a)

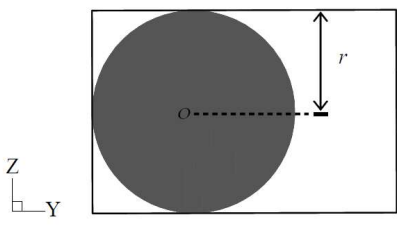

(b)

Fig. 7 An arbitrary polyhedral block and its maximum inscribed sphere. 

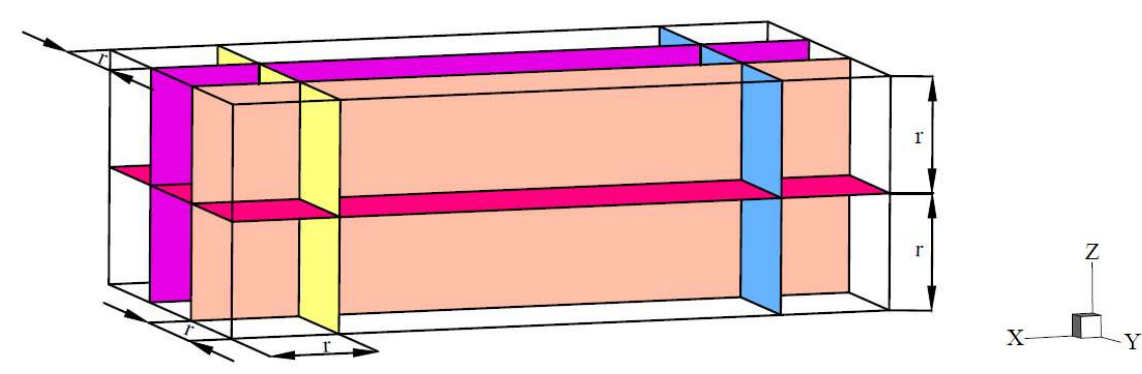

(a)
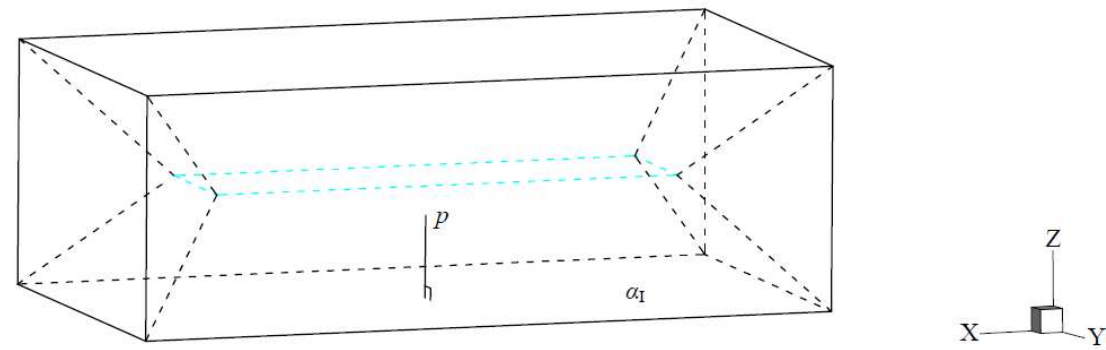

(b)

Fig. 8 An element is divided into sub-polyhedral blocks on the basis of the maximum inscribed sphere; where (a) the inner domain of the element according to $r$; (b) sub-polyhedral blocks and the point $p$ are shown.

Note that the new concept of dividing elements with the radius of a maximum inscribed sphere often results in an inner domain at the center of the element as presented in Fig. 8(b), especially for an arbitrary polyhedron and this phenomenon can be observed in Fig. 9. As described above, the point in this area is not defined by the distance potential function. In accordance with the basic assumption of the discrete element method, the embedded distance between contact couples is strictly controlled by the value of the normal contact stiffness. Consequently, the overlapping volume cannot extend to the core of the element because only a small penetration is allowed during the DEM computation, and the singular area without the definition of distance potential is not of significance for the solution procedure. 


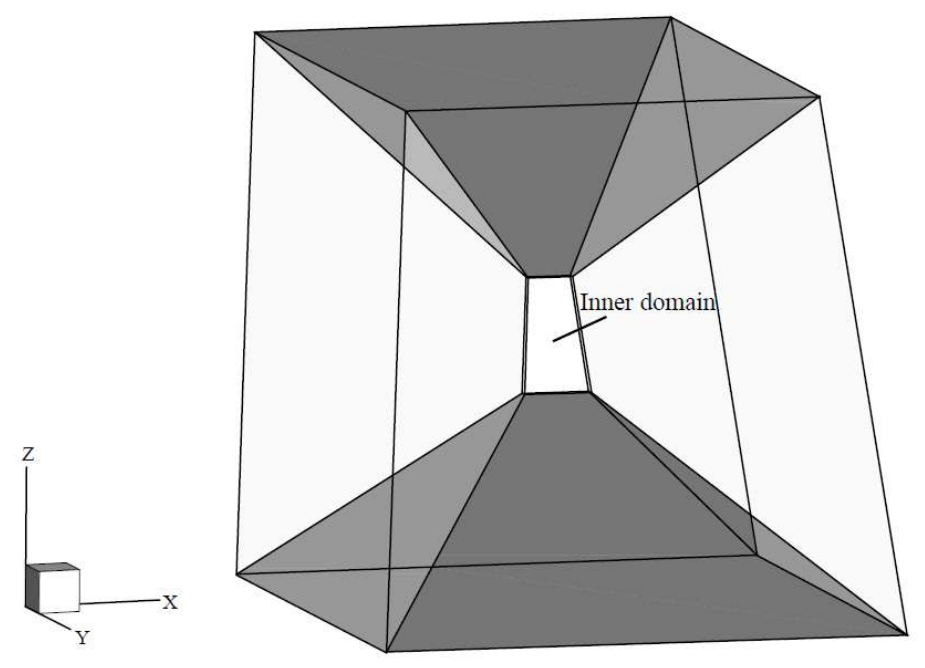

Fig. 9 Inner domain for an arbitrary polyhedron.

The merit of this definition lies in the approach of the approximated calculation of the contact interaction. As the normalized partition of the penetrated distance is applied in Eq. (5), the distance potential value is achieved without an influence of the element shape as presented in Fig. 10. Moreover, this new definition can be used in an arbitrarily convex polyhedron, because the principle of the distance potential function holds good universally. The constant of distribution gradient is also indicated in Fig. 11. The normal contact stiffness of different boundaries of the element is in accordance with the actual condition. As a result, it can avoid errors both in the calculation of potential and normal contact force as discussed in section 2.2 .

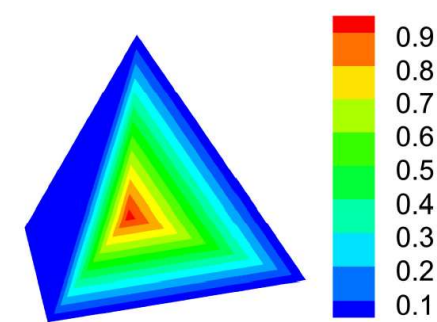

(a)

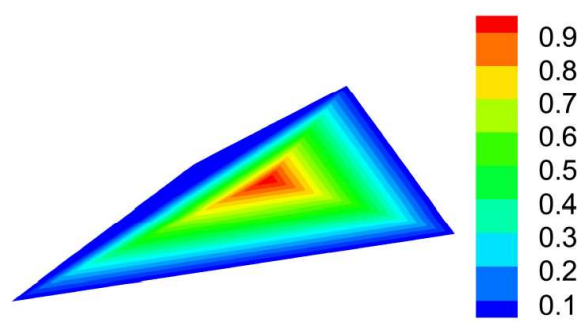

(b)

Fig. 10 The distance potential distribution in tetrahedral elements as in (a) a regular tetrahedron; (b) an arbitrary tetrahedron. 


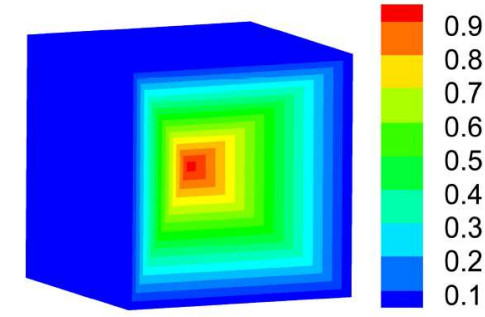

(a)

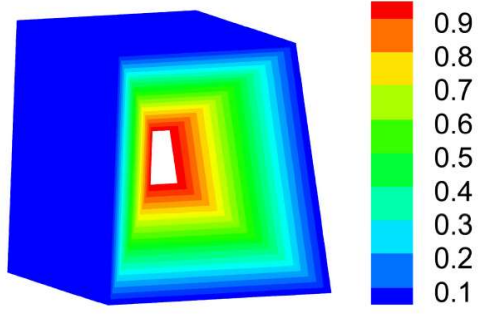

(b)

Fig. 11 The distance potential distribution in hexahedral elements as in (a) a cube; (b) an arbitrary hexahedron.

\subsection{Normal contact force}

In this section, the calculation process of the normal contact force is considered as based on Eq. (3) but with the newly defined distance potential function.

As illustrated in Fig. 12, two polyhedral blocks $\beta_{t}$ and $\beta_{c}$ are in contact. Normal contact force between this contact couples can be described as an integration of the distance potential function over the boundaries of the overlapping volume.

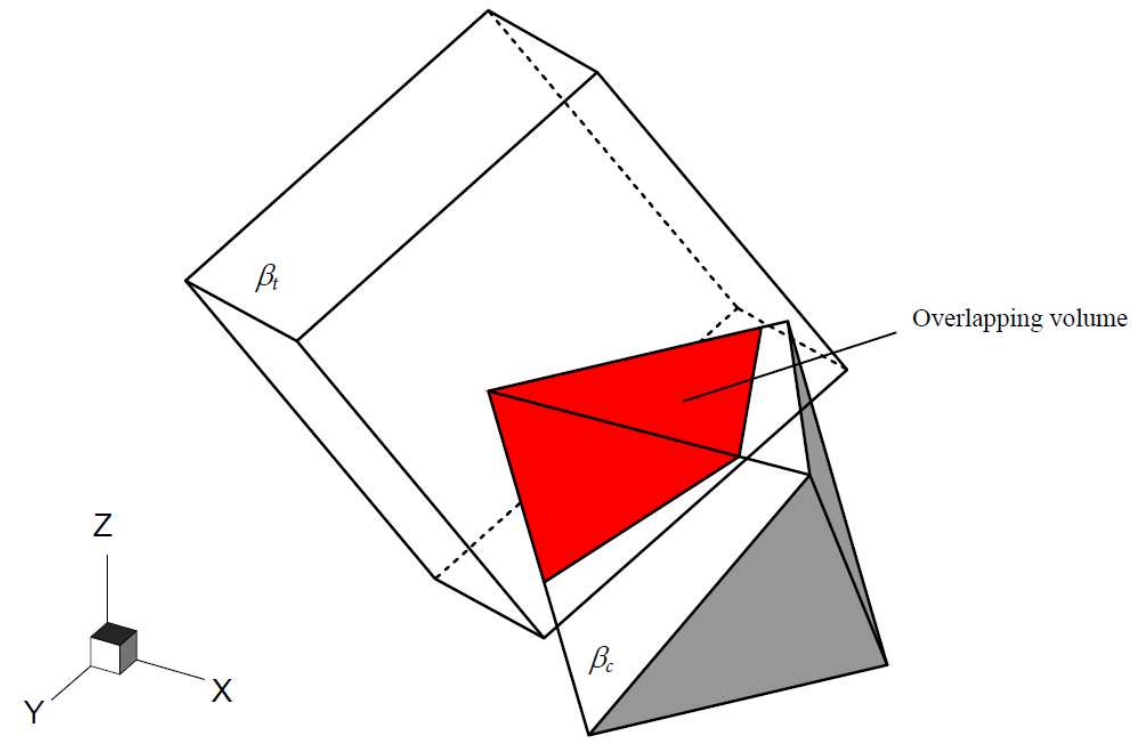

Fig. 12 Two contact polyhedrons and their overlapping volume.

In summary, two steps are listed as follows for the determination of the boundary surfaces:

Step 1. Determine the intersection surface $S$ among the plane of the base of $\beta_{c}$ and the target subpolyhedron of $\beta_{t}$ as indicated in Fig. 13 .

$$
S=\left\{S_{1}, S_{2} \ldots, S_{\mathrm{n}}\right\},
$$


where $S_{1}, S_{2} \ldots, S_{\mathrm{n}}$ are the nodes of the intersection surface $S$.

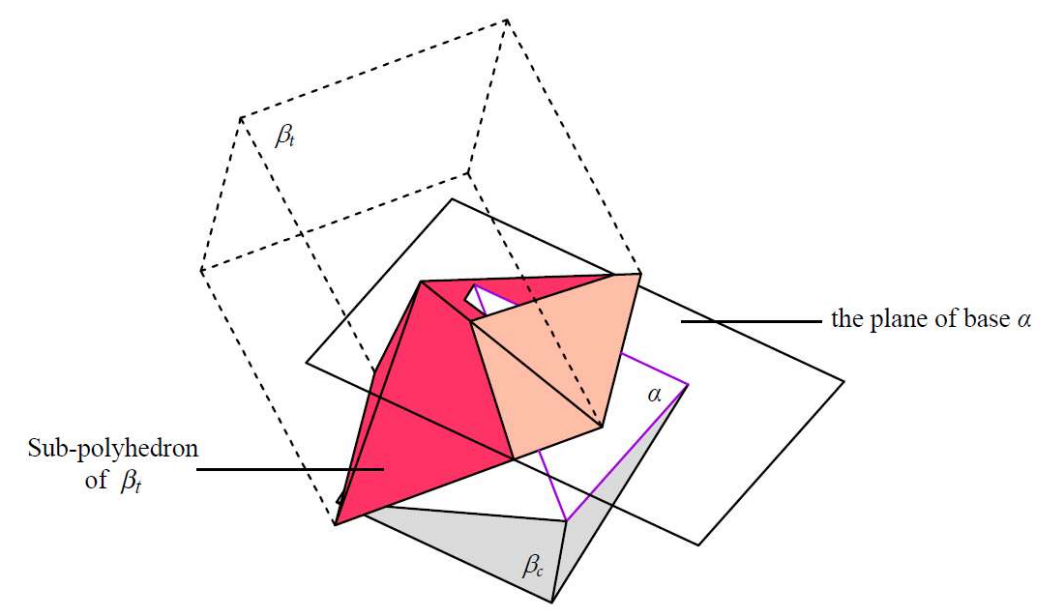

(a)

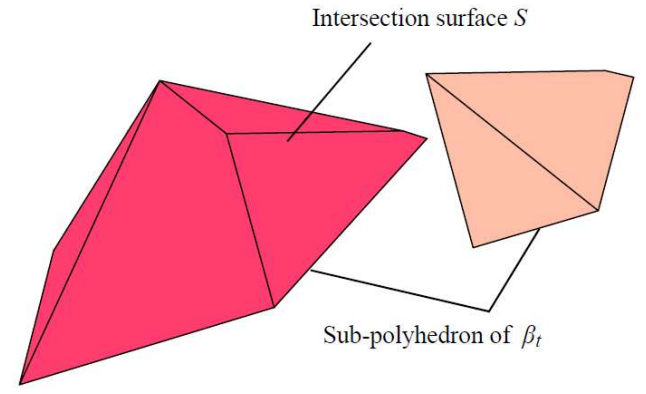

(b)

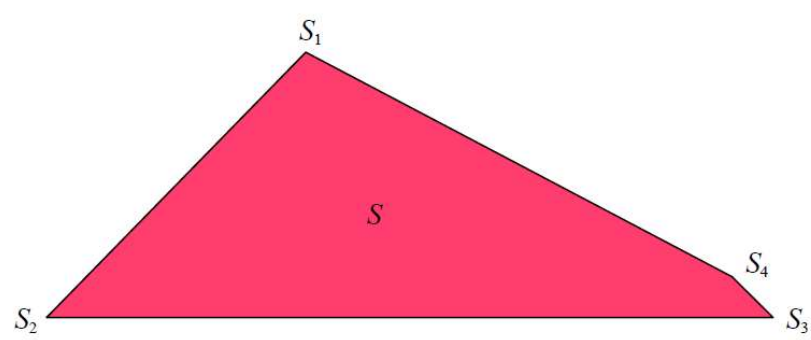

(c)

Fig. 13 Intersection surface $S$ between the plane base $\alpha$ of $\beta_{c}$ and a sub-polyhedron of $\beta_{t}$, showing (a) the sub-polyhedron of $\beta_{t}$ and the plane of $\alpha$ of $\beta_{c} ;(\mathrm{b})$ the sub-polyhedron of $\beta_{t}$ is separated into two parts by the plane of $\alpha$; (c) the intersection surface $s$ is determined by the subpolyhedron of $\beta_{t}$ and the plane of $\alpha$.

Step 2. Determine the intersection polygon $B$ defined by the surface $S$ and the base $\alpha$ of $\beta_{c}$ shown in Fig. 14.

$$
B=\left\{B_{1}, B_{2} \ldots, B_{n}\right\}
$$


where $B_{1}, B_{2} \ldots, B_{n}$ are the nodes of the polygon $B$.

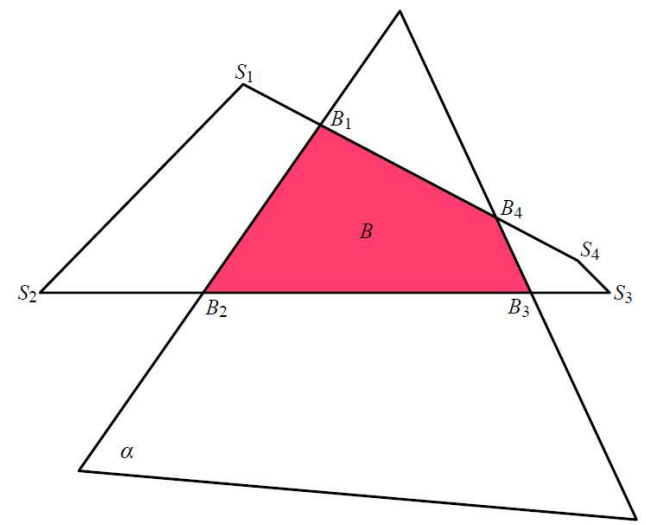

Fig. 14 The intersection polygon $B$ between the surface $S$ and the base $\alpha$.

Then the value of the distance potential at every node of the interaction polygon $B$ is presented as:

$$
\varphi_{d}\left(B_{1}\right), \varphi_{d}\left(B_{2}\right) \ldots, \varphi_{d}\left(B_{n}\right)
$$

The calculation is performed over the polygonal surface $B$. For simplicity, two assumptions are generated as base upon the regularities of the distribution of the distance potential function in the polyhedron: (i) The local coordinate system $(x, y)$ is established on the boundary surfaces, respectively. (ii) The distance potential distribution on the intersection surface $B$ has a linear variation and the value of the point over the surface $B$ is represented as:

$$
\varphi_{d}\left(x_{i}, y_{i}\right)=A_{1} x_{i}+A_{2} y_{i}+A_{3},
$$

where $x_{i}$ and $y_{i}$ are the local coordinates of the point on the polygonal surface $B$.

Parameters $A_{1}, A_{2}$ and $A_{3}$ can be expressed as:

$$
\begin{gathered}
A_{1}=\left[\varphi_{d}\left(B_{1}\right)\left(y_{3}-y_{2}\right)+\varphi_{d}\left(B_{2}\right)\left(y_{3}-y_{1}\right)+\varphi_{d}\left(B_{3}\right)\left(y_{1}-y_{2}\right)\right] /|J|, \\
A_{2}=\left[\varphi_{d}\left(B_{1}\right)\left(x_{3}-x_{2}\right)+\varphi_{d}\left(B_{2}\right)\left(x_{1}-x_{3}\right)+\varphi_{d}\left(B_{3}\right)\left(x_{2}-x_{1}\right)\right] /|J|, \\
A_{3}=\left[\varphi_{d}\left(B_{1}\right)\left(x_{2} y_{3}-x_{3} y_{2}\right)+\varphi_{d}\left(B_{2}\right)\left(x_{1} y_{3}-x_{3} y_{1}\right)+\varphi_{d}\left(B_{3}\right)\left(x_{1} y_{2}-x_{2} y_{1}\right)\right] /|J|,
\end{gathered}
$$

where $\left(x_{1}, y_{1}\right),\left(x_{2}, y_{2}\right)$ and $\left(x_{3}, y_{3}\right)$ are the local coordinates of $B_{1}, B_{2}$ and $B_{3}$, respectively.

Thus the normal contact force over the polygonal surface $B$ is:

$$
\boldsymbol{f}_{n, B}=\boldsymbol{n}_{B} k_{n} \iint \varphi_{d}(x, y) d x d y,
$$

where $\boldsymbol{n}_{B}$ is the outward unit vector of the polygonal surface $B, k_{n}$ is the normal penalty parameter. 
And the moments contributed by the contact normal force are:

$$
\begin{aligned}
& \boldsymbol{M}_{x, B}=\boldsymbol{n}_{B} k_{n} \iint \varphi_{d}(x, y) y d x d y, \\
& \boldsymbol{M}_{y, B}=\boldsymbol{n}_{B} k_{n} \iint \varphi_{d}(x, y) x d x d y .
\end{aligned}
$$

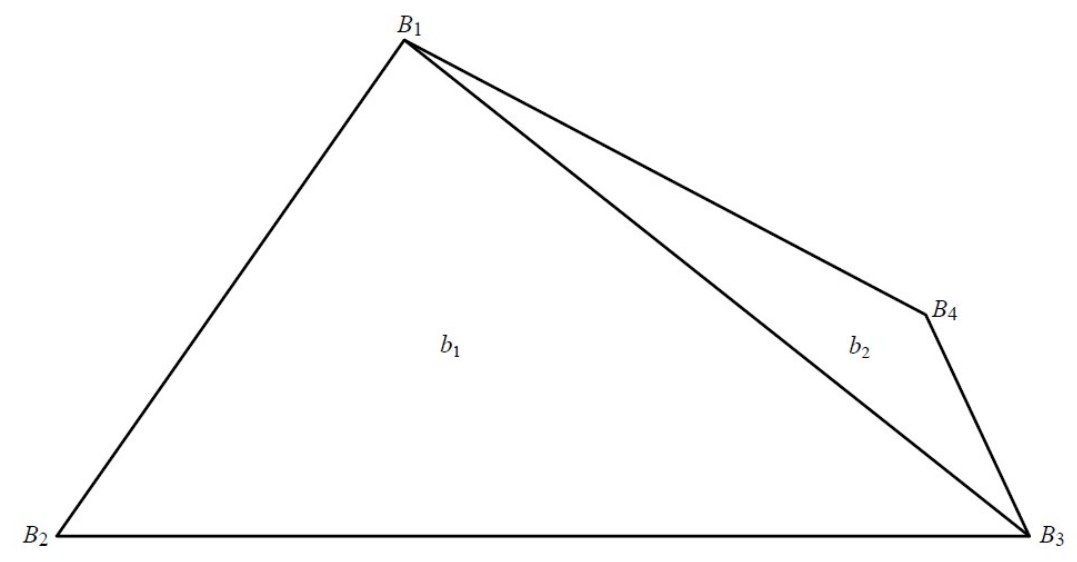

Fig. 15 The polygonal intersection surface $B$ is divided into triangles by the nodes.

The integration is performed by dividing the polygonal surface into triangles $b_{i}$ as shown in Fig. 15, then summating the total contact force over the triangular surfaces.

Note that the integration is computationally expensive over the triangular surfaces when there is a large number of elements and the shape of integral domain is arbitrary. Therefore, a natural coordinate system $(\eta, \zeta)$ located in every triangular surface is established for simplifying the calculation and improving computational efficiency.
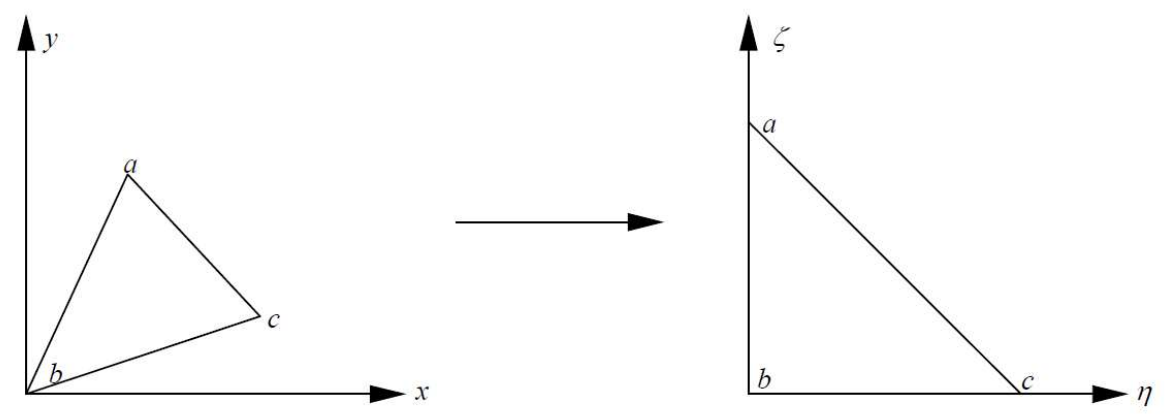

Fig. 16 Transition from the local coordinate system $(x, y)$ to natural coordinate system $(\eta, \zeta)$.

The local coordinate system and natural coordinate system shown in Fig. 16 are related through well-defined mappings:

$$
x(\eta, \zeta)=\sum_{i=1}^{3} N_{i}(\eta, \zeta) x_{i},
$$




$$
y(\eta, \zeta)=\sum_{i=1}^{3} N_{i}(\eta, \zeta) y_{i}
$$

where $N_{i}(\eta, \zeta)$ is the shape function expressed as:

$$
N_{1}(\eta, \zeta)=1-\eta-\zeta, \quad N_{2}(\eta, \zeta)=\zeta, \quad N_{3}(\eta, \zeta)=\eta, \quad(\eta, \zeta) \in(0,1)
$$

Therefore Eq.(9) can be described as:

$$
\varphi_{d}(x, y)=\varphi_{d}(\eta, \zeta)=\left(A_{1} x_{31}+A_{2} y_{31}\right) \eta+\left(A_{1} x_{21}+A_{2} y_{21}\right) \zeta+A_{1} x_{1}+A_{2} y_{1}+A_{3},
$$

where $x_{21}=x_{2}-x_{1}, y_{21}=y_{2}-y_{1}, x_{31}=x_{3}-x_{1}, y_{31}=y_{3}-y_{1}$.

The Eqs.(13), (14) and (15) can be rewritten as:

$$
\begin{gathered}
\boldsymbol{f}_{n, B}=\sum_{i=1}^{m} \boldsymbol{n}_{B} k_{n} \iint_{b_{i}} \varphi_{d}(x, y) d x d y=\sum_{i=1}^{m} \boldsymbol{n}_{B} k_{n} \iint_{b_{i}} \varphi_{d}(\eta, \zeta) d \eta d \zeta, \\
\boldsymbol{M}_{x, B}=\sum_{i=1}^{m} \boldsymbol{n}_{B} k_{n} \iint_{b_{i}} \varphi_{d}(x, y) y d x d y=\sum_{i=1}^{m} \boldsymbol{n}_{B} k_{n} \iint_{b_{i}} \varphi_{d}(\eta, \zeta)|J| d \eta d \zeta, \\
\boldsymbol{M}_{y, B}=\sum_{i=1}^{m} \boldsymbol{n}_{B} k_{n} \iint_{b_{i}} \varphi_{d}(x, y) x d x d y=\sum_{i=1}^{m} \boldsymbol{n}_{B} k_{n} \iint_{b_{i}} \varphi_{d}(\eta, \zeta)|J| d \eta d \zeta,
\end{gathered}
$$

where $m$ is the number of the divided triangular surfaces, $|J|$ is the Jacobi determinant of coordinate transformation, $|J|=\left|\begin{array}{ll}\frac{\partial x}{\partial \eta} & \frac{\partial y}{\partial \eta} \\ \frac{\partial x}{\partial \zeta} & \frac{\partial y}{\partial \zeta}\end{array}\right|$.

On the basis of Eq.(19), Eqs.(20), (21) and (22) can be simplified as:

$$
\begin{gathered}
\boldsymbol{f}_{n, B}=\sum_{i=1}^{m} \boldsymbol{n}_{B} k_{n}\left(\frac{C_{1}}{6}+\frac{C_{2}}{6}+\frac{C_{3}}{2}\right), \\
\boldsymbol{M}_{\eta, B}=\sum_{i=1}^{m} \boldsymbol{n}_{B} k_{n}\left(\frac{C_{1}}{12}+\frac{C_{2}}{24}+\frac{C_{3}}{6}\right), \\
\boldsymbol{M}_{\zeta, B}=\sum_{i=1}^{m} \boldsymbol{n}_{B} k_{n}\left(\frac{C_{1}}{24}+\frac{C_{2}}{12}+\frac{C_{3}}{6}\right) .
\end{gathered}
$$

Parameters $C_{1}, C_{2}, C_{3}$ are expressed as:

$$
\begin{gathered}
C_{1}=\left[A_{1}\left(x_{2}-x_{1}\right)+A_{2}\left(y_{2}-y_{1}\right)\right]|J|, \\
C_{2}=\left[A_{1}\left(x_{3}-x_{1}\right)+A_{2}\left(y_{3}-y_{1}\right)\right]|J|, \\
C_{3}=\left[A_{1} x_{1}+A_{2} y_{1}+A_{3}\right]|J| .
\end{gathered}
$$

The action position of the normal contact force in the natural coordinate system can be determined 
by:

$$
\left\{\begin{array}{l}
\eta_{n}=\frac{M_{\eta, B}}{f_{n, B}} \\
\zeta_{n}=\frac{M_{\zeta, B}}{f_{n, B}}
\end{array}\right.
$$

Analogously, the normal contact force and moment between the contact couples are decomposed into a summation at each base surface of the overlapping volume.

\subsection{Tangential contact force}

Munjiza (Munjiza et al. 1995) improved the calculating the algorithm of normal contact interaction and developed a simplified potential contact force method. However, the influence of the tangential contact force is not considered in his method. In this section, a precise computational algorithm for the tangential contact force is developed by the displacement increment method to revise this flaw in the FDEM approach.

The main difficulty of calculating the tangential contact force is the accurate determination of the tangential direction at each time step. As analyzed in section 3.2, contact interaction is decomposed as an integration over each boundary surface. Therefore, the measurement for tangential contact force can be generated in two different ways.

One possible way is to use an approximation of the algorithm for the normal contact force described in section 3.2. The tangential displacement increment and tangential force are implemented as components on the boundary surfaces (Yan et al. 2015; Yan and Zheng 2016). For brevity, the value of the total tangential contact force at step $i$ is denoted by

$$
\boldsymbol{f}_{s}^{i}=\sum_{j=1}^{n_{s}}\left(\boldsymbol{f}_{s}^{i-1}+k_{s} \cdot \Delta \boldsymbol{\delta}_{s, t}^{i}\right)_{j},
$$

where $n_{s}$ is the number of the boundary surfaces, $\boldsymbol{f}_{s}^{i}$ is the tangential contact force at step $i$, $\Delta \boldsymbol{\delta}_{s, t}^{i}$ is the tangential increment displacement of each surface, and $k_{s}$ is the tangential penalty parameter. The tangential direction is defined as verticality of the normal contact force on each surface.

It is worthwhile to note that large computer memory (RAM) is necessary to record both the normal and tangential force and displacement increments at each step for all boundary surfaces. More importantly, this method could cause excess normal force. As indicated in Fig. 17(a) and (b), block $\beta_{\mathrm{I}}$ impacts block $\beta_{\mathrm{II}}$ in a direction opposite that of $\mathrm{Z}$ axis. Initially blocks $\beta_{\mathrm{I}}$ and $\beta_{\mathrm{II}}$ are stacked in 
such way that they contact, but there are no overlap and contact force between them. The calculation of the contact interaction is presented in Fig. 17(c). It is observed that the tangential forces $\boldsymbol{f}_{t, a c}$ and $\boldsymbol{f}_{t, a b}$ have components along the normal direction which will affect the computation of the normal contact force. And this can be a source of error.

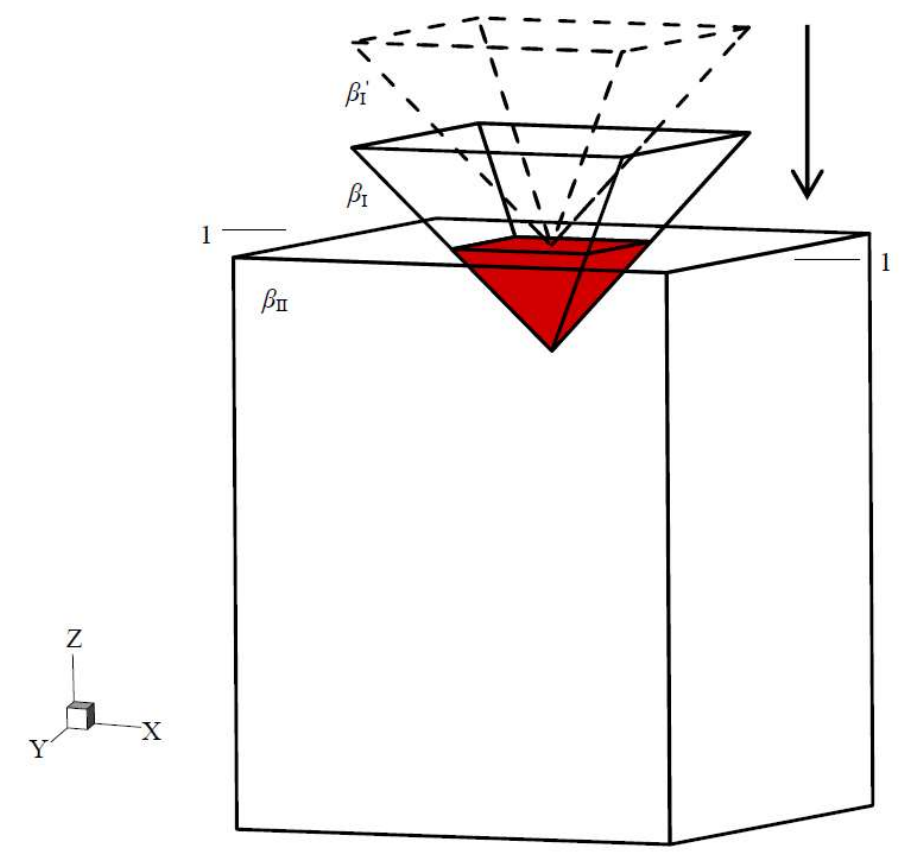

(a)

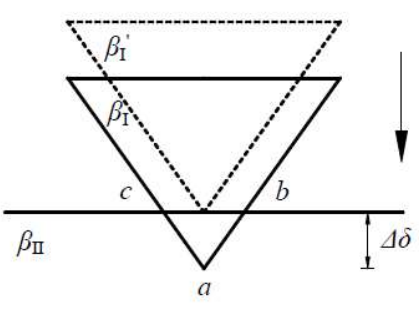

(b)

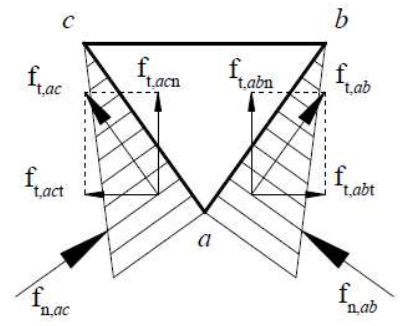

(c)

Fig. 17 The calculation of a tangential contact force on each boundary surface, where (a) block $\beta_{\mathrm{I}}$ penetrates $\beta_{\mathrm{II}}$ along $\mathrm{Z}$ axis; (b) the calculation model of cross section 1-1; (c) the contact interactions in $1-1$.

An alternative approach is illustrated in Fig. 18. The calculation for tangential force is achieved by following the solution of the total normal contact force. The value of the tangential force is determined by the total tangential increment displacement. The direction of the tangential force is perpendicular to the total normal contact force and the loading position is almost identical with the total normal contact 
force. Hence excessive computer memory requirements and the errors analyzed for the first method can be completely eliminated.

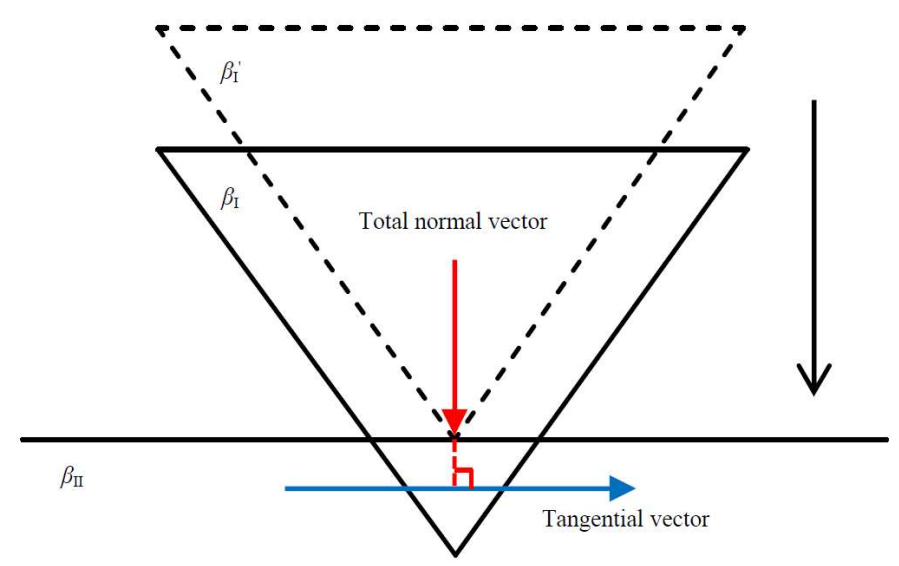

Fig. 18 Calculation of tangential contact force based on the total tangential increment displacement.
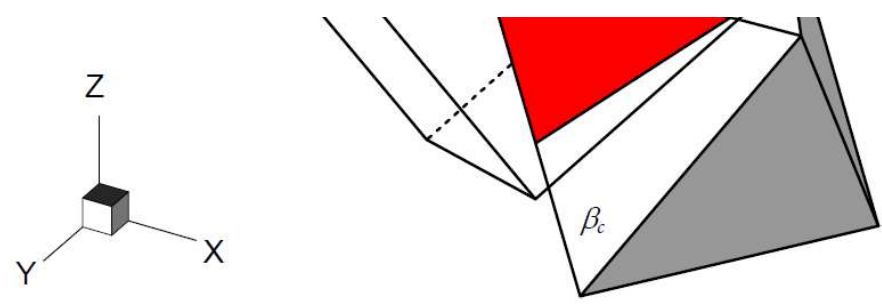

Im. To achieve the with respect to the

Fig. 12 at step $i$ is considered as

$$
v^{i}=v_{c}^{i}-v_{t}^{i}+\omega_{c}^{i} \times r_{c}^{i}-\omega_{t}^{i} \times r_{t}^{i}
$$

where $\boldsymbol{v}_{c}^{i}$ and $\boldsymbol{v}_{t}^{i}$ are the translational velocities of blocks $\beta_{c}$ and $\beta_{t}$ at step $i$, respectively; $\boldsymbol{\omega}_{c}^{i}$ and $\boldsymbol{\omega}_{t}^{i}$ stand for the angular velocities of blocks $\beta_{c}$ and $\beta_{t}$, respectively. Then the incremental tangential displacement $\Delta \boldsymbol{\delta}_{s}^{i}$ is:

$$
\Delta \boldsymbol{\delta}_{s}^{i}=\Delta \boldsymbol{s}^{i}-\left(\Delta \boldsymbol{s}^{i} \cdot \boldsymbol{n}_{n}\right) \cdot \boldsymbol{n}_{n}=\left[\boldsymbol{v}^{i}-\left(\boldsymbol{v}^{i} \cdot \boldsymbol{n}_{n}\right) \cdot \boldsymbol{n}_{n}\right] \cdot \boldsymbol{\Delta t},
$$

where $\Delta \boldsymbol{s}^{i}$ is the incremental displacement between $\beta_{c}$ and $\beta_{t}$, and $\boldsymbol{n}_{n}$ is the unit direction vector of total normal contact force.

The tangential contact force is updated as:

$$
\boldsymbol{f}_{s}^{i}=r \times \boldsymbol{f}_{s}^{i-1}+\Delta \boldsymbol{f}_{s}^{i},
$$

where $r$ is the rotation matrix that rotates the normal vector from step $i-1$ to the normal vector at the current step $i, \Delta \boldsymbol{f}_{s}^{i}$ is the incremental tangential contact force expressed as:

$$
\Delta \boldsymbol{f}_{s}^{i}=k_{s} \cdot \Delta \boldsymbol{\delta}_{s}^{i}
$$

The magnitude of the tangential force is checked with the maximum possible value $\left(f_{s}\right)_{\max }$ 
defined by Coulomb-type friction law as:

$$
\left(f_{s}\right)_{\max }=f_{n} \cdot \tan \varphi_{\mu}+C,
$$

where $\varphi_{\mu}$ is the maximum static friction angle, $C$ is the cohesion force. If the absolute value of $f_{s}$ is larger than $\left(f_{s}\right)_{\max }, f_{s}$ is set to be equal to $\left(f_{s}\right)_{\max }$.

The tangential contact moment is given by

$$
\boldsymbol{M}_{s}^{i}=\boldsymbol{f}_{s}^{i} \times \gamma_{s},
$$

where $\gamma_{s}$ is the vector from the force $\boldsymbol{f}_{s}$ load position to the centroid of the element.

\section{Contact detection algorithm}

As mentioned in the previous sections, the proposed method is applied to deal with the motion of a system consisting of arbitrary convex polyhedra. In general, the simulation model contains a large amount of individual blocks with different sizes. An efficient contact detection algorithm is crucial since it can affect the computational efficiency of the discrete element method. One of the most common contact detection algorithm is the NBS (no binary search) firstly developed by Munjiza (Munjiza and Andrews 1998). It is a linear calculation algorithm, in other words, both the total CPU time and RAM requirements are proportional to the number of blocks. Because the method is insensitive to the spatial distribution of the elements, it displays a high effectiveness during the contact detection process both in dense and loose packs of elements with a uniform size. However, this method cannot be used for the problems of non-uniform blocks. As the system is discretized according to the size of the largest block, contact detections for small blocks are also performed even though they are far away from each other. To overcome this problem, Munjiza (Munjiza et al. 2006) developed the linear search algorithm for the system comprised of different sizes of particles and proposed the multi-step MR algorithm (MMR).

In this work, an improved search algorithm is proposed for non-uniform polyhedral blocks depending upon the basic idea of MMR. The approach is performed by dividing the elements into different groups according to their sizes:

Group 1: Value of the maximum circumradius for the first group blocks $d_{1}: D / \gamma<d_{1} \leq D$,

Group 2: Value of the maximum circumradius for the second group blocks $d_{2}: D / \gamma^{2}<d_{2} \leq D / \gamma$,

Group n: Value of the maximum circumradius for the last group blocks $d_{n}: d_{n} \leq D / \gamma^{n-1}$, where $D$ is the maximum circumradius among all the blocks, $\gamma>1$. 


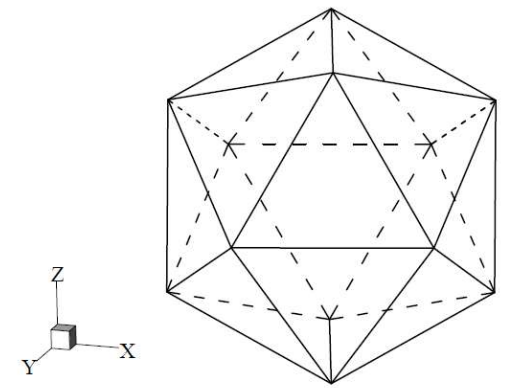

(a)

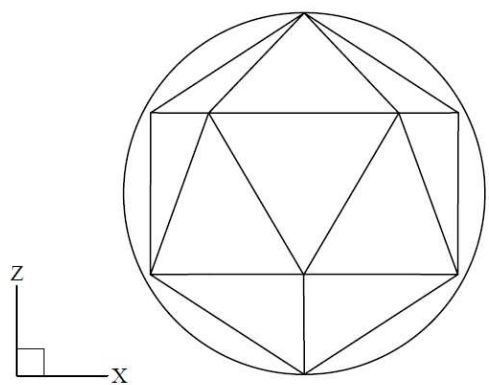

(b)

Fig. 19 Spherical bounding box of an icosahedron.

Then the process of the contact search algorithm can be performed in $\mathrm{n}$ steps as follows.

Step 1. As shown in Fig. 19, all of the elements are bounded by spherical boxes and they are mapped into the calculation space which is discretized into cells according to the maximum value of $d_{1}$ in Fig. 20 . Each block can only be mapped into a cell based on its centroid, as explained in the following formulations:

$$
\begin{aligned}
& x_{k}=1+\operatorname{int}\left(\frac{x_{\text {cent }}-x_{\min }}{l}+0.5\right), \\
& y_{k}=1+\operatorname{int}\left(\frac{y_{\text {cent }}-y_{\min }}{l}+0.5\right), \\
& z_{k}=1+\operatorname{int}\left(\frac{z_{\text {cent }}-z_{\text {min }}}{l}+0.5\right),
\end{aligned}
$$

where $x_{c e n t}, y_{c e n t}$ and $z_{c e n t}$ are the current coordinates of the centroids in the global coordinate system, $x_{k}, y_{k}$ and $z_{k}$ are the coordinates of the centroids when the elements are mapped into the cell, $l=\max \left\{d_{1}\right\}$.

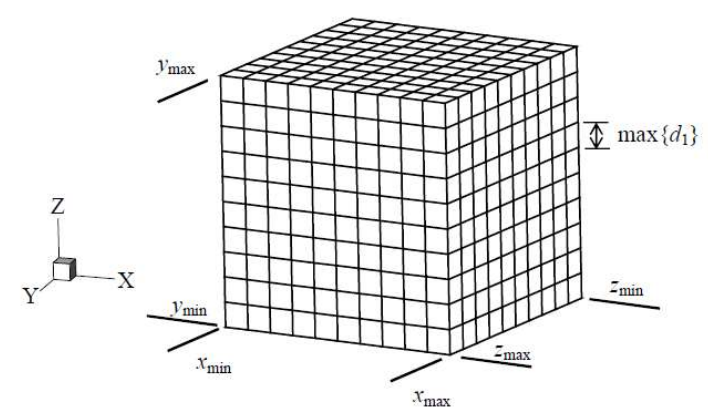

(a)

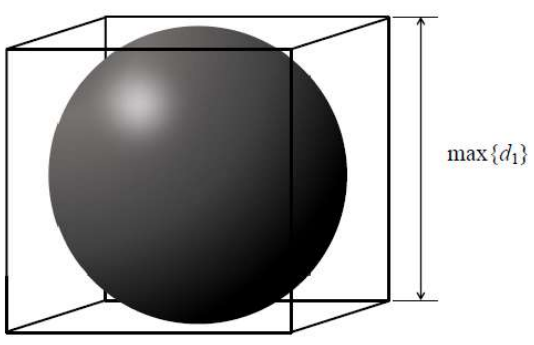

(b)

Fig. 20 Space divided into identical cells which are large enough to contain the first group element. 
The contact detection is performed for the first group elements. The detection of contact for the first group elements is circulated as a direct check from either central or neighboring cells as shown in Fig. 21. Discrete elements mapped into the cells can be determined as contact couples only if the contact condition is met:

$$
l_{c} \leq l_{\max },
$$

where $l_{c}$ is the distance between the centroids of the contact couples. And $l_{\max }$ is the contact distance: $l_{\max }=l_{1 \max }+l_{2 \max }, l_{1 \max }, l_{2 \max }$ are the longest distances between the vertexes and centroids of the two contact elements, respectively.

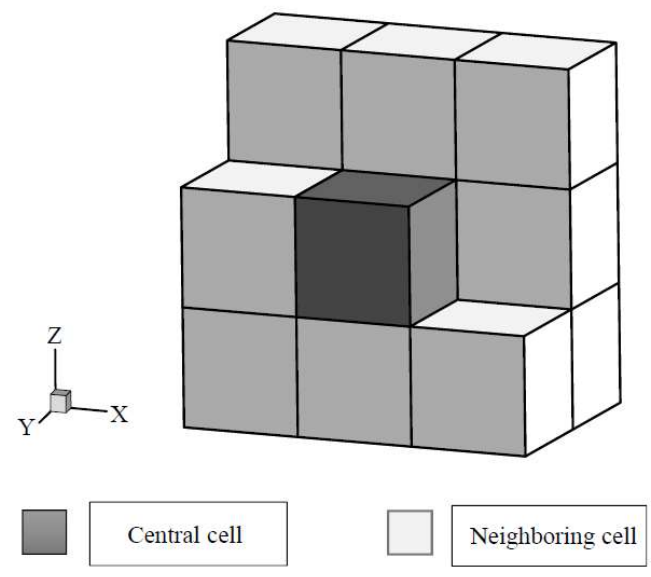

(a)
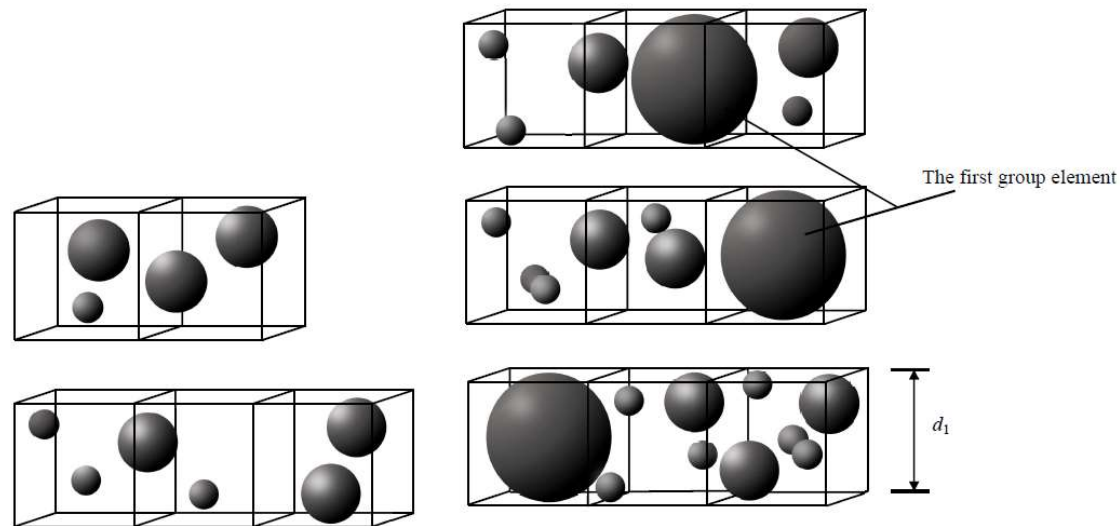

(b)

Fig. 21 Contact detection check cells, where (a) the blank cell is the central cell and the shaded elements are neighboring cells, (b) all the blocks are mapped into the cells and the contact detection for the first group elements is performed.

The contact elements for the first group elements can be obtained respectively. 
Step 2: The first group elements are removed from the system and all the remaining elements are mapped into the space which is divided by $d_{2}$ as shown in Fig. 22. Contact detection is performed between the elements of group 2 and remaining group elements with the method in step 1 . Thus the contact couples for the second group elements can be detected.

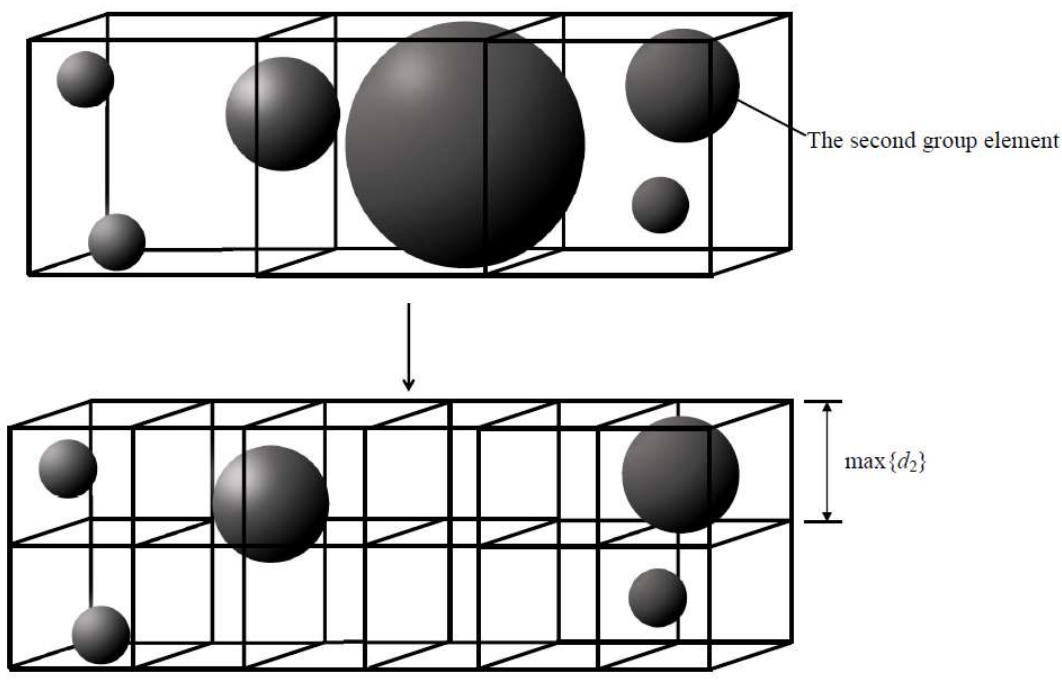

Fig. 22 The first group elements are removed and space is divided into identical cells according to $d_{2}$

Step n: By analogy, in this step only elements in group $\mathrm{n}$ remain in the system and the contact detection is carried out between these elements.

With the new contact search algorithm, the size of the cells in each step is approximated to fit the detection performed elements. The coordinates of the element mapped into the cells can reflect the actual distribution law of the elements and it can greatly improve the accuracy of contact detection for the system comprised of blocks with different sizes.

\section{Verification and Application}

In this section, several numerical examples are presented to illustrate the accuracy of the newly proposed method. Firstly, the robustness is tested by five standard problems, including an impact simulation, a numerical friction experiment, a joints structure effects on the sliding rock mass, a pillar impact, and a block accumulation. Then the full simulation of this method is performed for a stability analysis of a wedge slope. The results are compared against existing numerical and experimental data in literature.

\subsection{Impact simulation between tetrahedral blocks}


The proposed method exhibits an accurate computation algorithm of the normal contact force. To verify its reliability, a test for an impact process is analyzed.

This numerical model is composed of two tetrahedral blocks $\beta_{\mathrm{I}}$ and $\beta_{\mathrm{II}}$ which are vertically arranged along the $\mathrm{Y}$ axis shown in Fig. 23. The density of blocks is $2000 \mathrm{~kg} / \mathrm{m}^{3}$ and the initial velocity of block A is set as $\boldsymbol{u}=0 \mathrm{~m} / \mathrm{s}$. The vertical acceleration due to the gravity is taken to be $10 \mathrm{~m} / \mathrm{s}^{2}$ in a direction opposite that of the $\mathrm{Y}$ axis, and the friction is neglected. In this simulation, large embedding is allowed with the application of a small penalty parameter for observation during the process of penetration. As a comparison, a test with the same setting is conducted by FDEM.
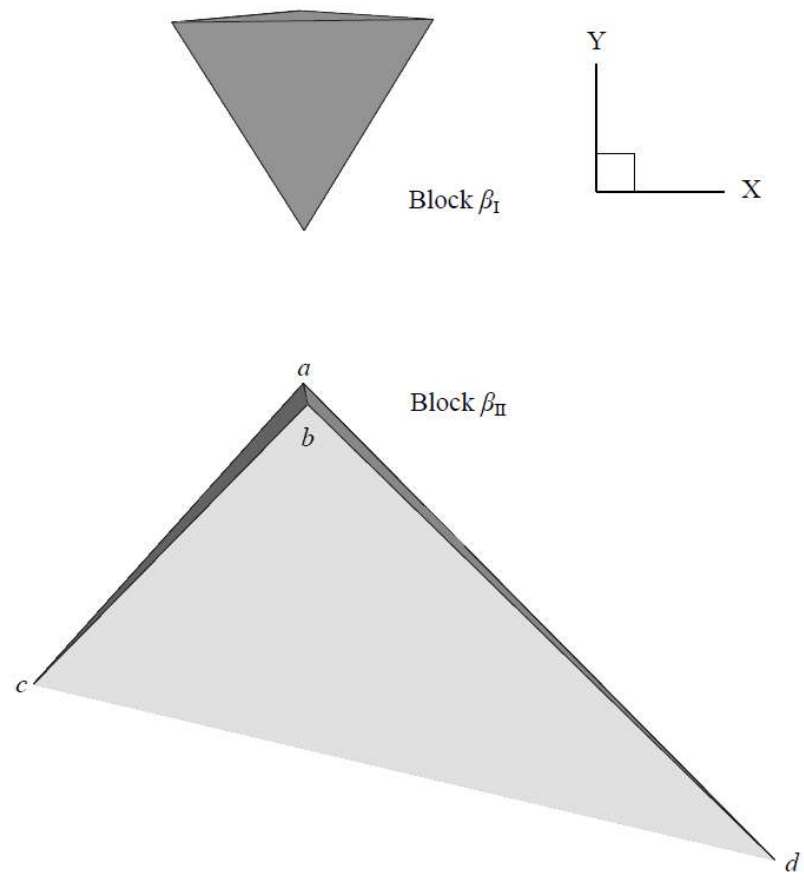

Fig. 23 Numerical model for the impact test.

Initially block $\beta_{\mathrm{I}}$ is free-falling towards $\beta_{\mathrm{II}}$. As $\beta_{\mathrm{I}}$ impacts the ridge $a-b$, the velocity decreases and converses to a standstill during the increment of the penetration. In this phase, the embedded volume between the contact couples achieves the maximum value simultaneously. Then A turns into a reverse motion and ultimately returns the original state for the negligible energy loss.

The predicted motion of block $\beta_{\mathrm{I}}$ with FDEM is illustrated in Fig. 24. It is worthwhile to notice that the track and the final position of block $\beta_{\mathrm{I}}$ are not compatible before and after the collision, and sideways deflection of block $\beta_{\mathrm{I}}$ can be observed in the simulation results. Because a sensitivity of the calculated normal contact force by Eq.(1) to the element shape, the simulation with FDEM is inconsistent with the physical visualization. 


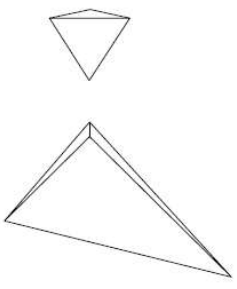

(a) $t=0 \mathrm{~s}$

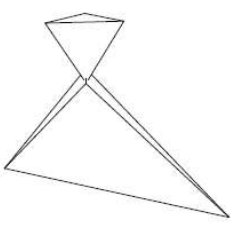

(b) $t=0.752 \mathrm{~s}$

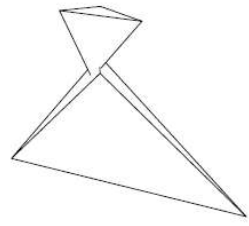

(c) $t=0.814 \mathrm{~s}$

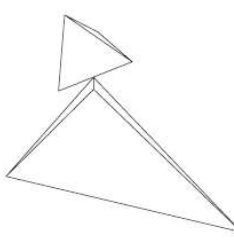

(d) $t=0.876 \mathrm{~s}$

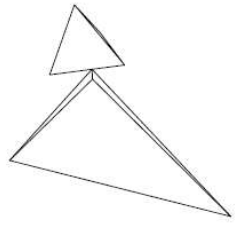

(e) $t=0.9 \mathrm{~s}$

Fig. 24 Predicted motion of block $\beta_{\mathrm{I}}$ by FDEM
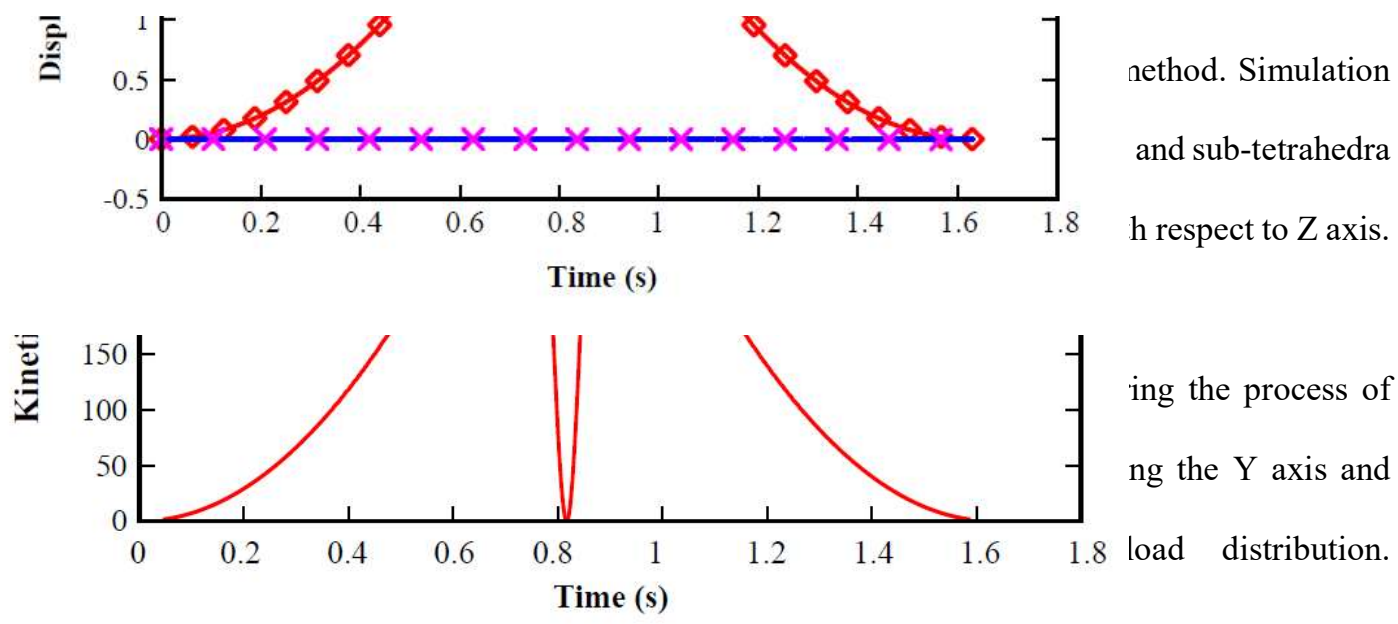

Fig. 27Fig. 27 shows the evolution of the energy conservation obtained by the proposed method. The reduction of kinetic energy is the consequence of the contact from $t=0.72 \mathrm{~s}$. The kinetic energy translates into potential energy due to the overlap in contact. Then the potential energy translate into the kinetic energy. The kinetic energy equals with the value at the time $t=0.72 \mathrm{~s}$, when the block $\beta_{\mathrm{I}}$ begins to move away from the block $\beta_{\mathrm{II}}$.

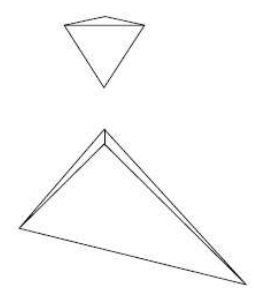

(a) $t=0 \mathrm{~s}$

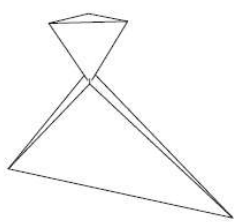

(b) $t=0.752 \mathrm{~s}$

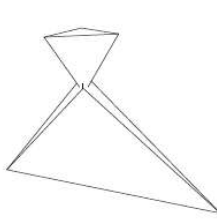

(c) $t=0.814 \mathrm{~s}$

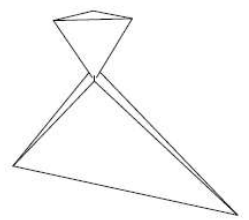

(d) $t=0.876 \mathrm{~s}$

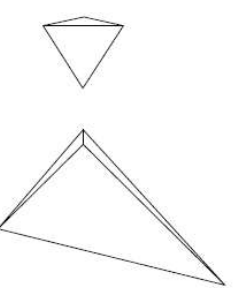

(e) $t=1.628 \mathrm{~s}$

Fig. 25 Predicted motion of block $\beta_{\mathrm{I}}$ by the newly proposed method. 


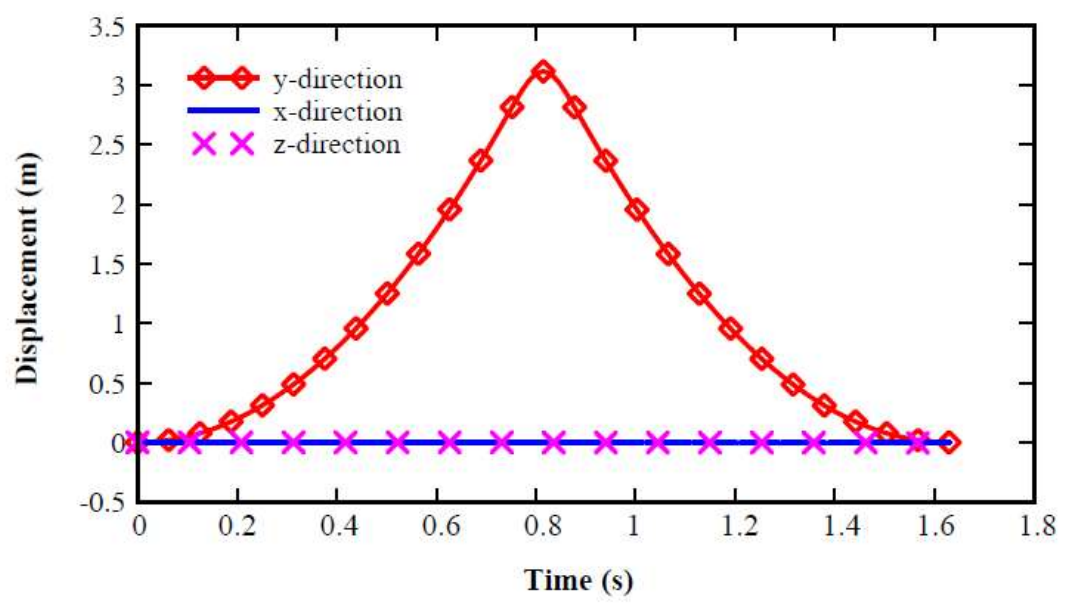

Fig. 26 Three displacement components of block $\beta_{\mathrm{I}}$ at different times.

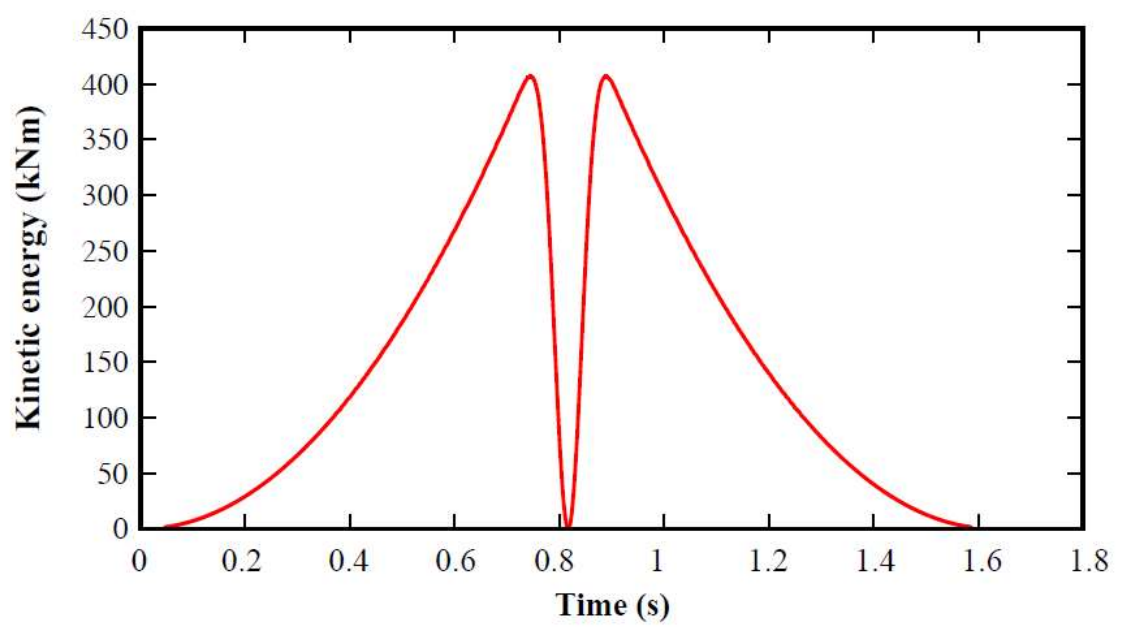

Fig. 27 Kinetic energy of block $\beta_{\mathrm{I}}$ as a function of time by the newly proposed method.

Based on the above discussion, the veracity of the proposed method in the calculation of the normal contact force is deduced. However, the tangential contact force has not been tested yet. Thus a test for the friction experiment is implemented in the next section.

\subsection{Test for the frictional experiment}

In this example, a friction test for a cuboid sliding on an inclined surface is simulated as a benchmark problem for this proposed method. The analytical formulas of the displacement and velocity for the block during the sliding process are given as follows:

$$
\boldsymbol{s}=\left\{\begin{array}{c}
\boldsymbol{u}_{0} \cdot t-\frac{1}{2}(g \sin \theta+g \cos \theta \cdot \mu) t^{2}, t \leq t_{1} \\
\boldsymbol{s}_{0}-\frac{1}{2}(g \sin \theta-g \cos \theta \cdot \mu) t^{2}, t>t_{1}
\end{array},\right.
$$




$$
\boldsymbol{u}= \begin{cases}\boldsymbol{u}_{0}-(g \sin \theta+g \cos \theta \cdot \mu) t, & t \leq t_{1} \\ (g \sin \theta+g \cos \theta \cdot \mu) t, & t \leq t_{1}\end{cases}
$$

where $\boldsymbol{u}_{0}$ and $\boldsymbol{s}_{0}$ are the initial velocity and displacement of the sliding block, respectively, $g$ stands for the gravity acceleration, $\theta$ represents the slope angle of inclined plane, $\mu$ is the friction coefficient of plane surface and $t_{1}=u_{0} /(g \sin \theta+g \cos \theta \cdot \mu)$.

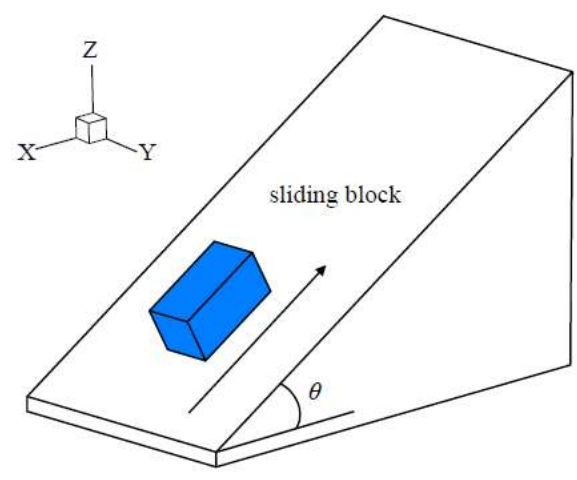

(a)

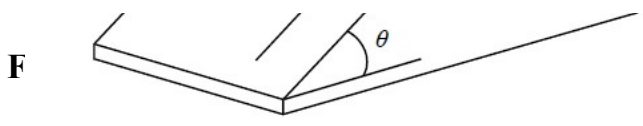

(a)

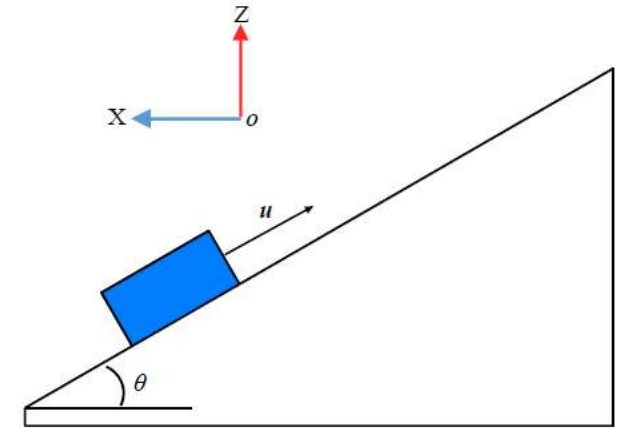

(b)

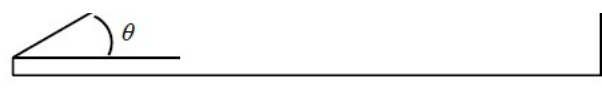

10

(b)

Fig. 28Fig. 28 presents the numerical model of this test. The material properties of the sliding block in the simulation are listed in Table 1.

Table 1 Material properties of the sliding block, test for the friction experiment.

\begin{tabular}{ccccc}
\hline $\begin{array}{c}\text { Density } \\
\left(\mathrm{kg} / \mathrm{m}^{3}\right)\end{array}$ & $\begin{array}{c}\text { Young's } \\
\text { Modulus }(\mathrm{Pa})\end{array}$ & Poisson ratio & $\begin{array}{c}\text { Normal stiffness } \\
(\mathrm{N} / \mathrm{m})\end{array}$ & $\begin{array}{c}\text { Tangential } \\
\text { stiffness }(\mathrm{N} / \mathrm{m})\end{array}$ \\
\hline 2500 & $2 \times 10^{8}$ & 0.167 & $1.5 \times 10^{8}$ & $1.2 \times 10^{8}$ \\
\hline
\end{tabular}

Initially the velocity of the sliding block is $\boldsymbol{u}_{0}=6 \mathrm{~m} / \mathrm{s}$, then the gravitational force drives the block to move along the dipping slope surface. Fig. 29 and Fig. 30 show the displacement and velocity of the sliding block with a friction coefficient $0.2,0.4$ and 0.6 , respectively. Results calculated by the proposed method agree well with the theoretical values. 


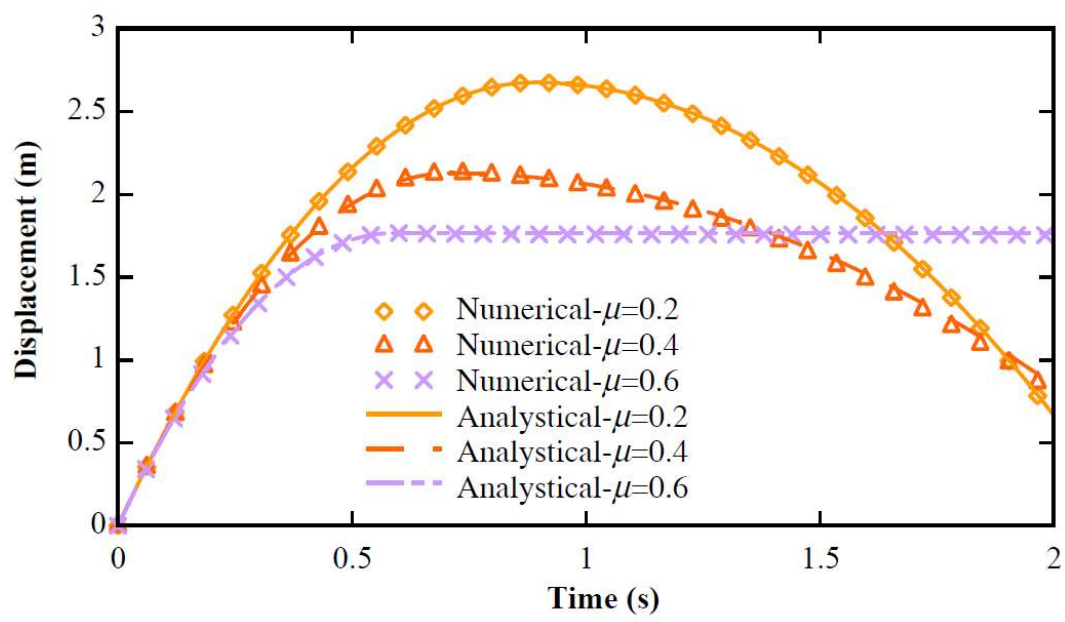

Fig. 29 Time evolution of the displacement of the sliding block for several friction coefficients as calculated by the proposed numerical method and the theoretical expression (41).

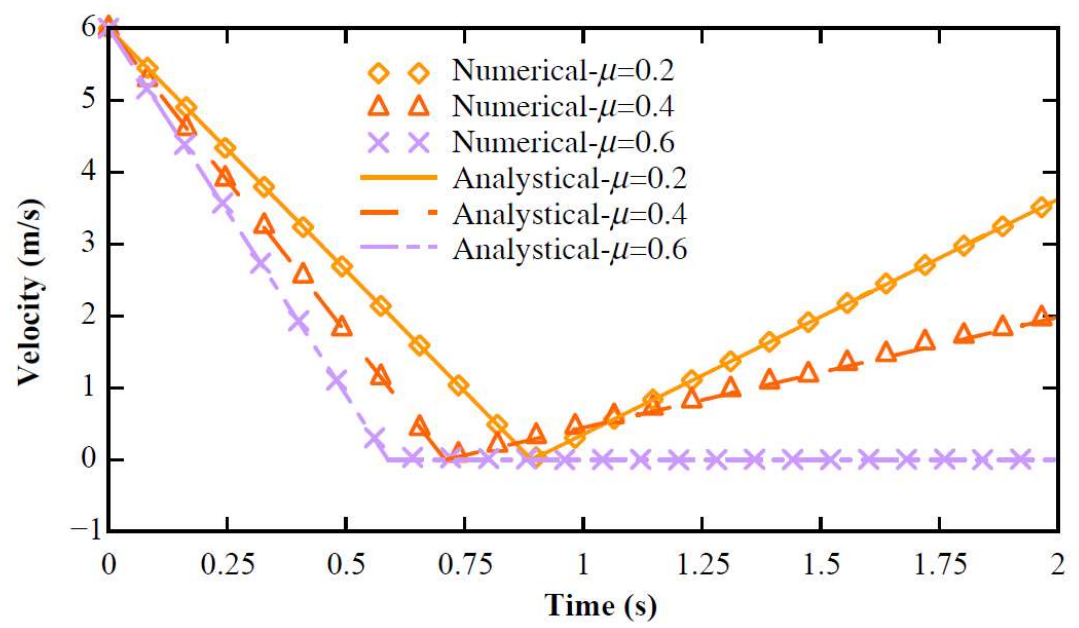

Fig. 30 Time evolution of the velocity of the sliding block for several friction coefficients as calculated by the proposed numerical method and the theoretical expression (42).

Fig. 31 shows the displacement time evolution for several normal penalty parameters. Excellent match is found between the numerical and the analytical predictions. 


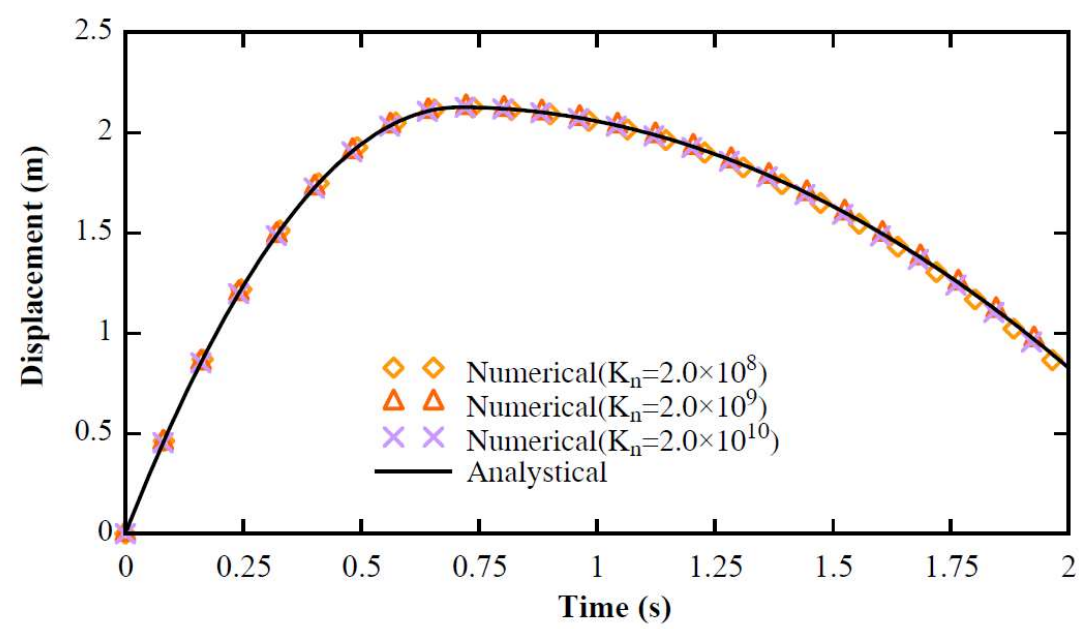

Fig. 31 Time evolution of the displacement of the sliding block for several normal penalty parameters as calculated by the proposed numerical method and the theoretical expression (41).

\subsection{Simulation for joints structure affecting a sliding rock mass}

The calculation of the tangential contact force is significantly dependent on the incremental tangential displacement. A major obstacle for an errorless computation of tangential contact force is the variation of the direction of tangential contact force within each time step. A method for this problem was presented in this work, and it is tested in a simulation of joints structure effects of a sliding rock mass. This simulation is based on the experiment pursued by $\mathrm{Li}$ ( $\mathrm{Li}$ et al. 2007). Our results are compared against the experiment data and their available numerical results.

The experiment is designed with five artificial slopes as shown in Table 2. Three different sizes of blocks are used in this example to form the slopes and their geometries are listed in Table 3.

Table 2 Models of five accumulation slopes with different sets of transfixion planes.

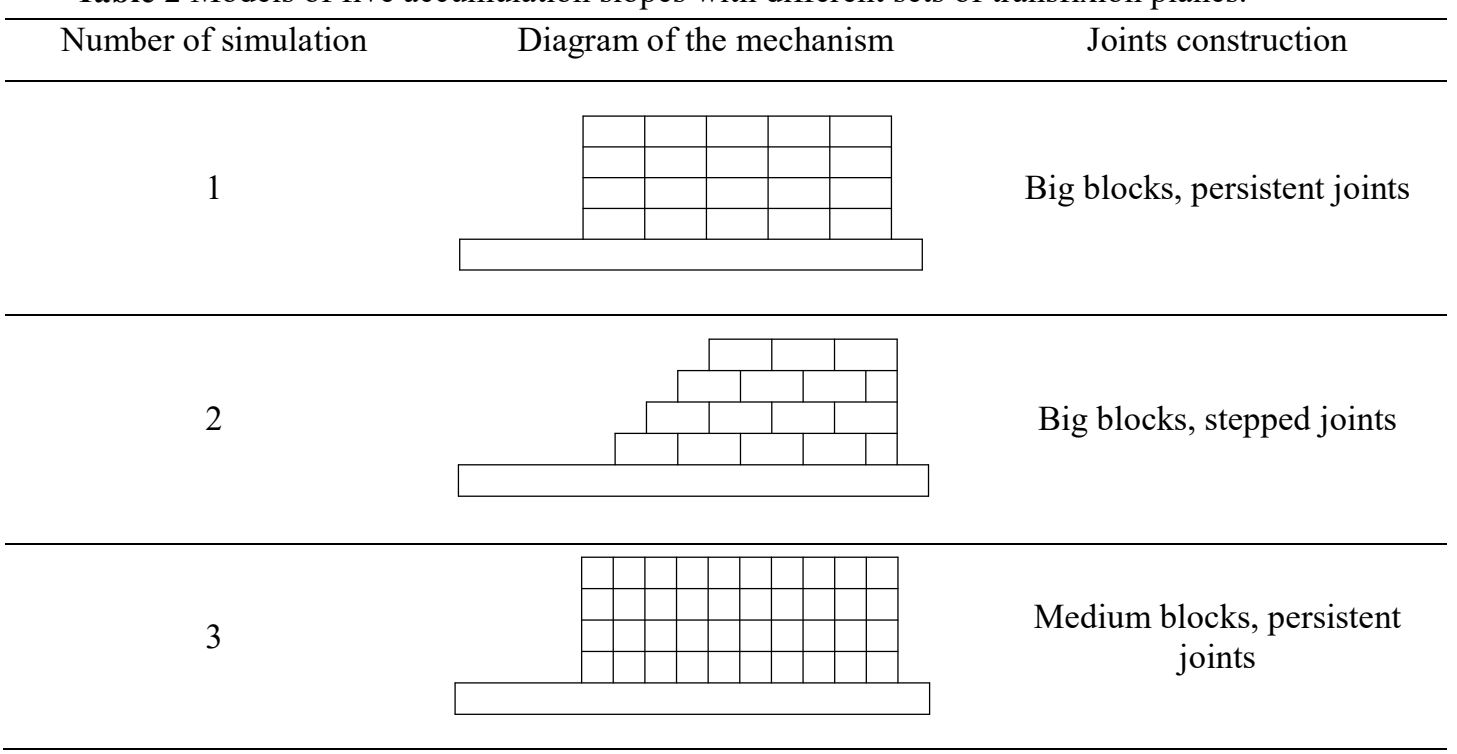




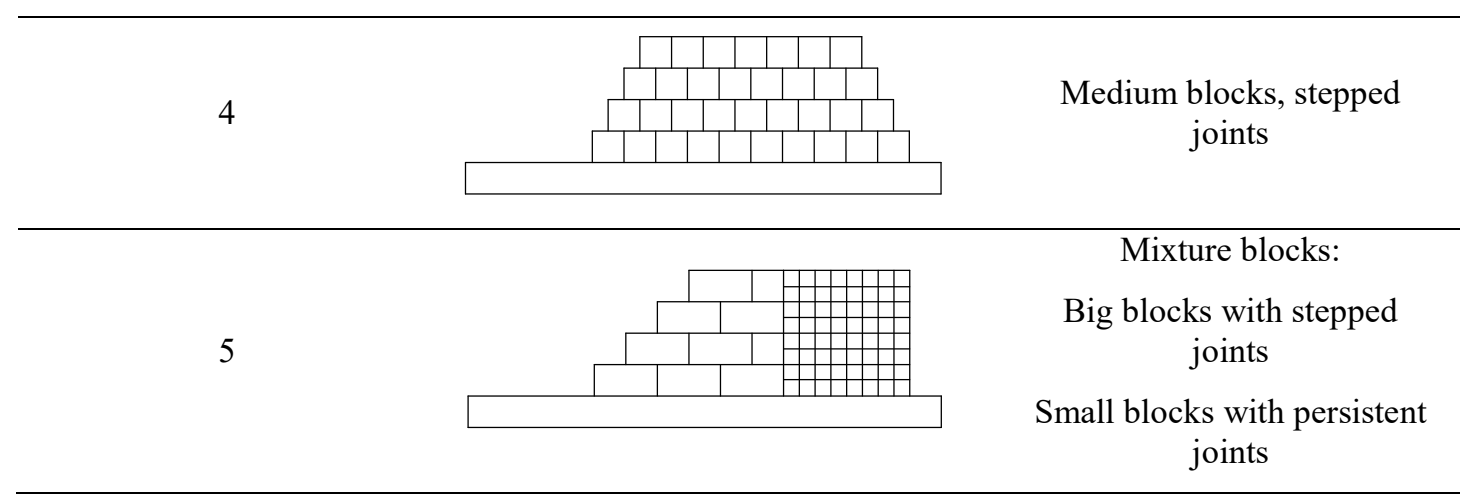

Table 3 The geometrical properties of three types of blocks.

\begin{tabular}{cccc}
\hline Block type & Length $(\mathrm{m})$ & Width $(\mathrm{m})$ & Height $(\mathrm{m})$ \\
\hline Big block & 0.2 & 0.1 & 0.1 \\
\hline Medium block & 0.1 & 0.1 & 0.1 \\
\hline Small block & 0.05 & 0.05 & 0.05 \\
\hline
\end{tabular}

In the experiment, five types of accumulation slopes are placed on a platform, respectively. The platform is turned counterclockwise with a constant angular velocity until the failure of the slope occurs.

The blocks have a density of $2000 \mathrm{~kg} / \mathrm{m}^{3}$ and a gravity acceleration of $9.81 \mathrm{~m} / \mathrm{s}^{2}$ was assumed. All the blocks and the platform have the same mechanical parameters: the friction coefficient is $\mu=0.4877$, the cohesion is $C=2.14 \mathrm{~Pa}$, the normal penalty parameter is $k_{n}=2.0 \mathrm{Gpa}$, and the tangential penalty parameter is $k_{s}=1.6 \mathrm{Gpa}$. The constant angular velocity of the platform is $0.03 \mathrm{rad} / \mathrm{s}$.

Table 4 shows the simulation results of the five types of accumulation slopes, presenting the failure behavior of each accumulation slope. The failure process of the fifth slope is exhibited in Fig. 32. The simulation results, including the failure modes and failure angles, are compared with the experimental data and numerical results by $\mathrm{Li}$ ( $\mathrm{Li}$ et al. 2007), as exhibited in Table 5. It can be observed that simulation results of the proposed method are in a good agreement with the existing experimental and numerical data. Hence the proposed method can deal with a tangential direction change in time. 
Table 4 The simulation results of five slopes.

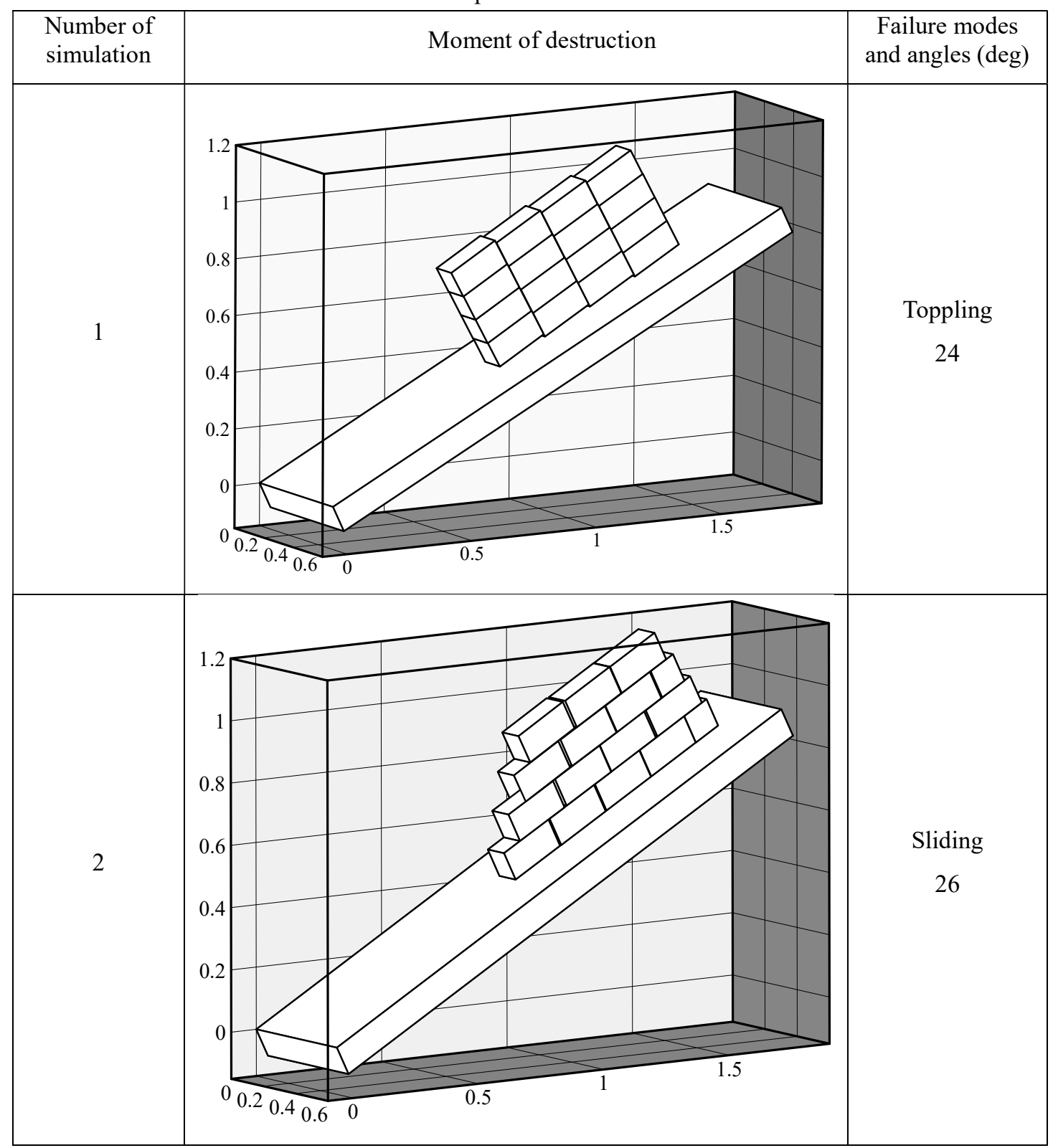




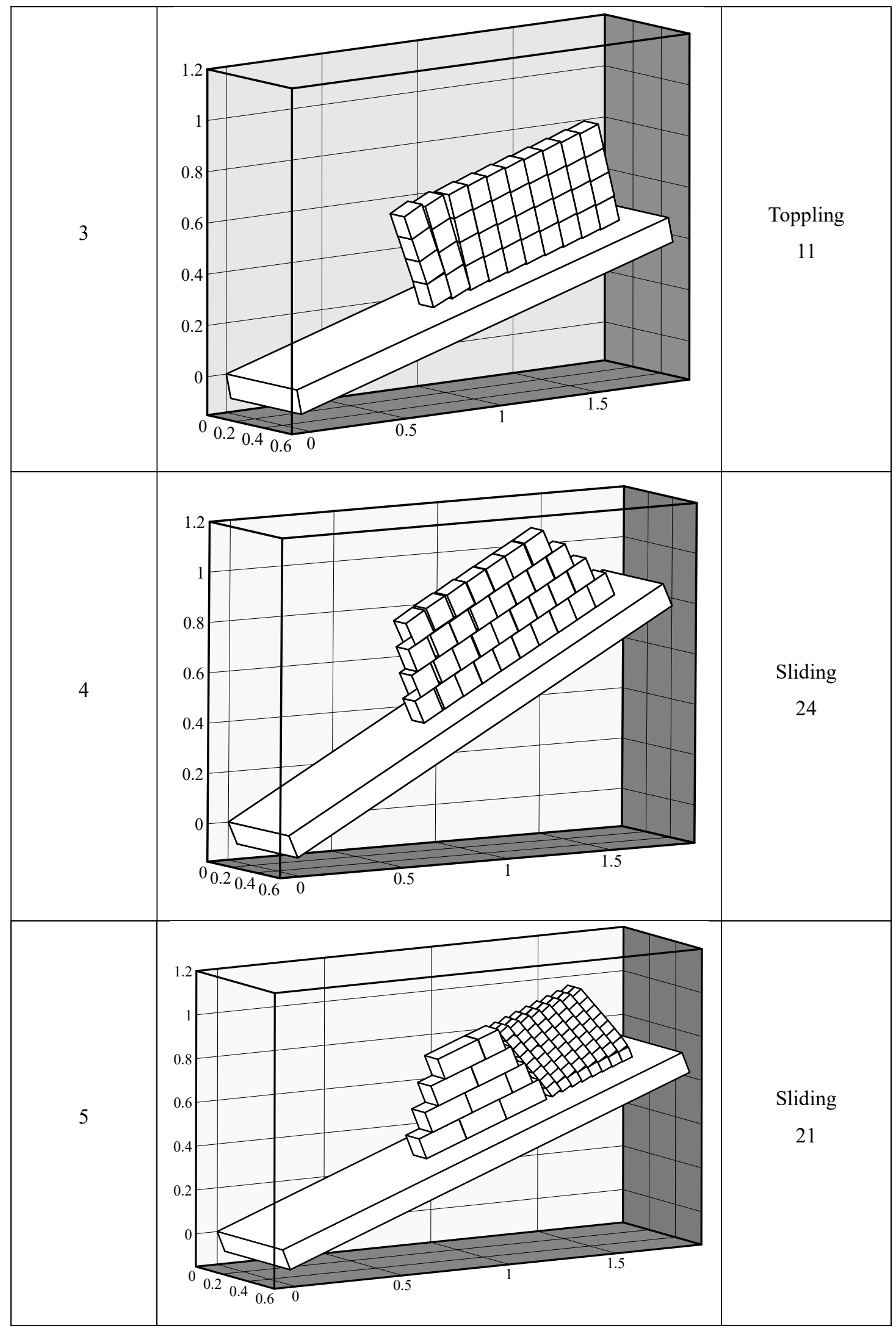




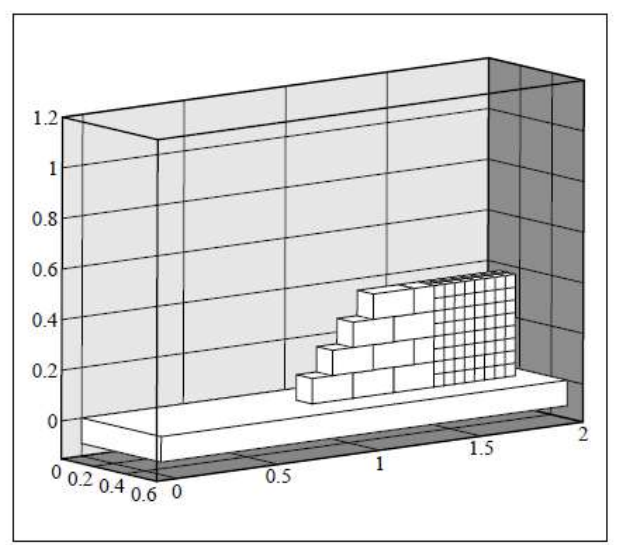

(a)

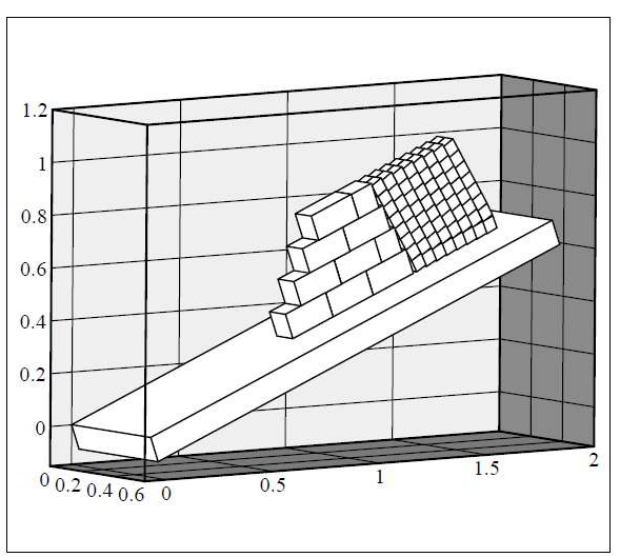

(c)

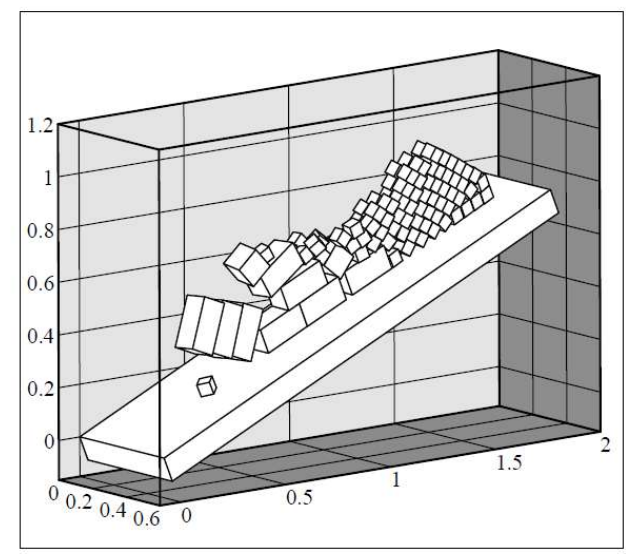

(e)

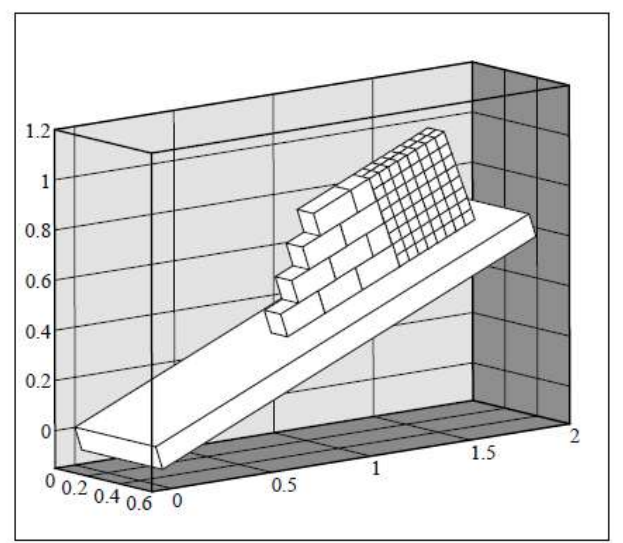

(b)

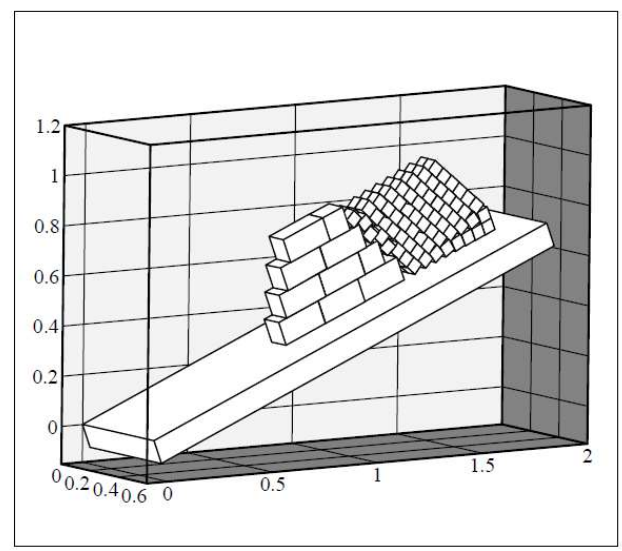

(d)

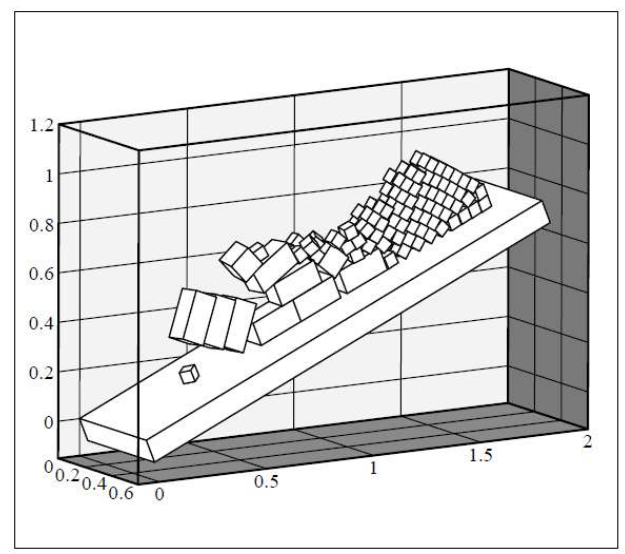

(f)

Fig. 32 Failure process of the fifth slope consists of the mixture blocks. (a) $t=0 \mathrm{~s}$; (b) $t=11.7 \mathrm{~s}$; (c)

$$
t=12.0 \mathrm{~s} ; \text { (d) } t=12.6 \mathrm{~s} \text {; (e) } t=13.0 \mathrm{~s} \text {; (f) } t=13.2 \mathrm{~s} \text {. }
$$

Table 5 Comparison of the current simulation results with experimental data and numerical results by $\mathrm{Li}(\mathrm{Li}$ et al. 2007).

\begin{tabular}{ccccccc}
\hline \multirow{2}{*}{ Failure mode } & 1 & 2 & 3 & 4 & 5 \\
\cline { 3 - 6 } & & Toppling & Sliding & Toppling & Sliding & Sliding \\
\hline Failure & The proposed method & 24.0 & 26.0 & 11.0 & 24.0 & 21.0 \\
\hline
\end{tabular}




\begin{tabular}{lcccccc}
\hline angle & \multicolumn{1}{c}{$(\mathrm{deg})$} \\
\cline { 2 - 6 } & $\begin{array}{c}\text { Experiment data by Li } \\
(\mathrm{deg})\end{array}$ & $22.1 \sim 24.2$ & $25.0 \sim 26.3$ & $9.8 \sim 11.6$ & $23.0 \sim 24.9$ & $19.5 \sim 21.8$ \\
\cline { 2 - 6 } & $\begin{array}{c}\text { Numerical result by Li } \\
(\mathrm{deg})\end{array}$ & 23.0 & 25.5 & 10.0 & 23.5 & 20.0 \\
\hline
\end{tabular}

\subsection{Impact simulation of a pillar}

An impact simulation for a pillar is employed to test the ability of the proposed method to deal with complex situations. The original configuration of the pillar is shown in Fig. 33. This calculation model is discretized by $0.5 \times 0.5 \times 0.5 \mathrm{~m}^{3}$ rectangular blocks. The initial velocity of the rigid projectile is set as $\boldsymbol{u}=-50 \mathrm{~m} / \mathrm{s}$ in $\mathrm{Y}$ direction. The blocks use the same density of $600 \mathrm{~kg} / \mathrm{m}^{3}$ and a gravity acceleration of $9.81 \mathrm{~m} / \mathrm{s}^{2}$ is assumed. The strength and cohesion of all interfaces between blocks are zero. The friction coefficient is 0.5 , the normal penalty parameter is $2 \mathrm{Gpa}$, and the tangential penalty parameter is $2 \mathrm{Gpa}$. The detection results are computed with the motion law discussed by Jin (Jin et al. 2011).
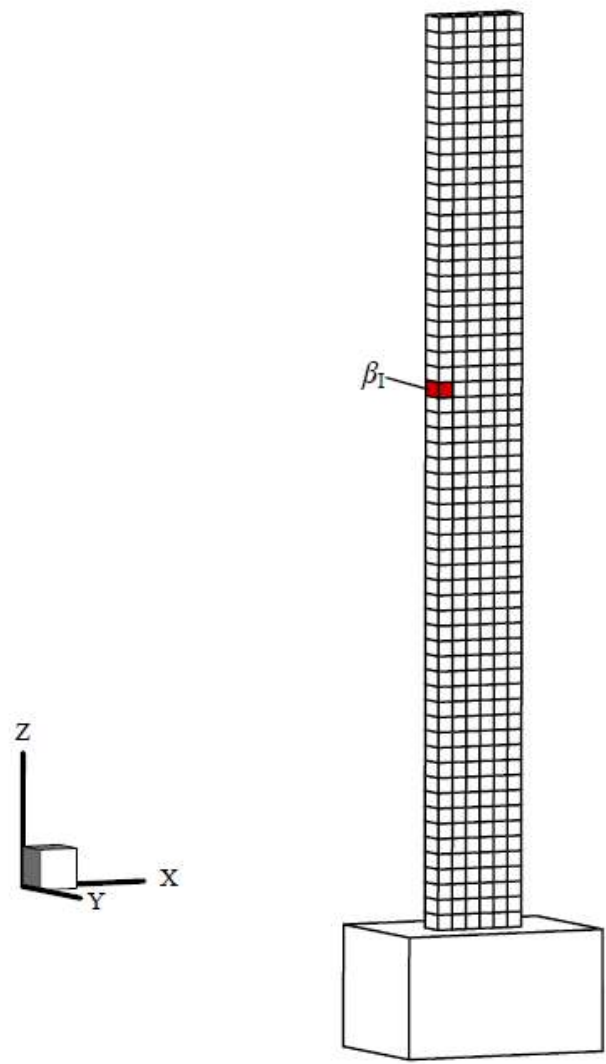

Fig. 33 Numerical model of the pillar impact case.

The predicted transient motion of the pillar is given in Fig. 34. The movement of the blocks is consistent with the analysis by Jin (Jin et al. 2011). As the projectile impacts the center of the pillar, two 
kinds of waves are generated. The first one is the compression wave formed by the impact, which propagate at the impact direction. As a result, the blocks in pillar near the impact point are extracted by the projectile. This results in an acceleration of these blocks. It can be observed that the front blocks fly forward and the blocks around the impacting point fly backward.

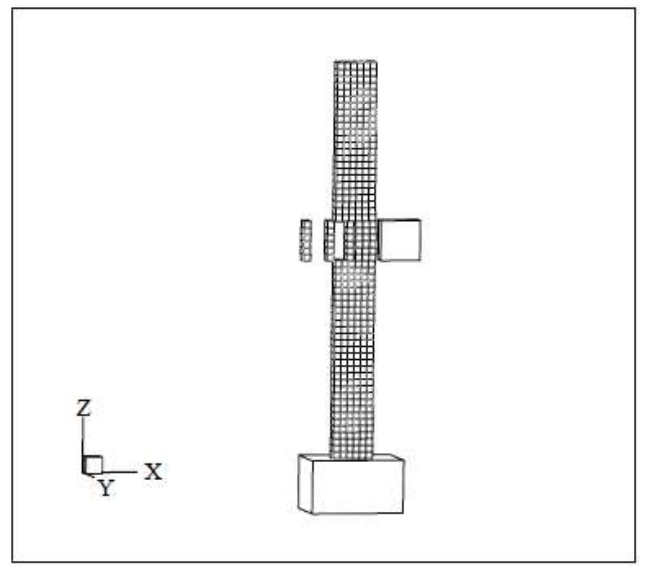

(a)

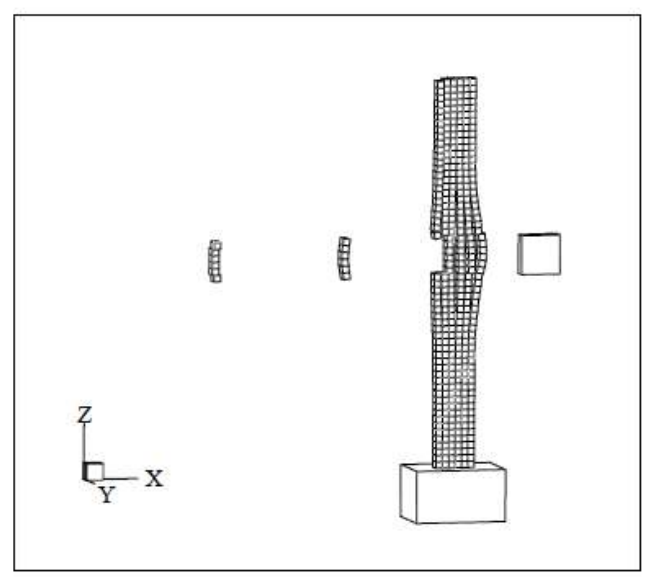

(c)

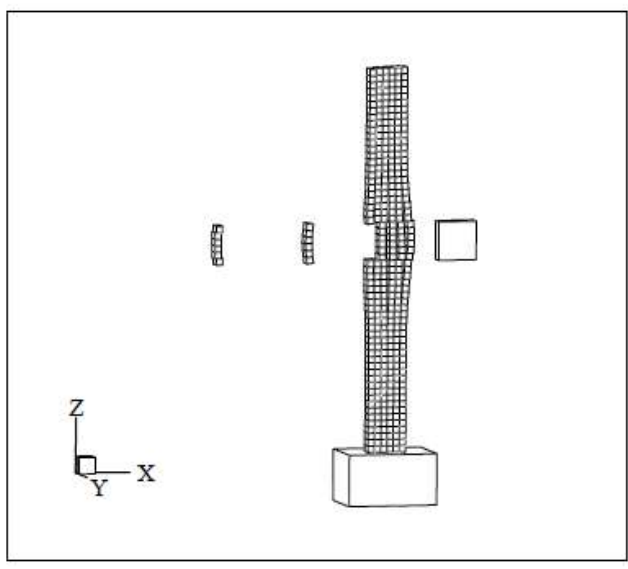

(b)

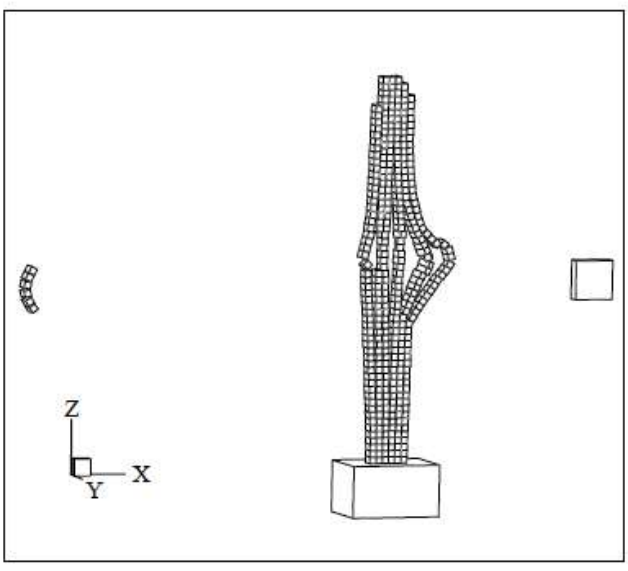

(d)

Fig. 34 Predicted motion of the pillar blocks at different times of (a) $t=0.03 \mathrm{~s}$; (b) $t=0.14 \mathrm{~s}$; (c) $t=$

$$
0.20 \mathrm{~s} \text { and (d) } t=0.72 \mathrm{~s} .
$$

Propagation of the pressure waves satisfy the standard wave equation, and the wave velocity $V$ can be calculated as follows:

$$
V=\sqrt{\frac{E}{\rho}},
$$

where $E$ is the elastic modulus, and $\rho$ is the density of the blocks. $E$ is assigned to all blocks, therefore the elastic modulus is obtained by Hooke's Law

$$
\boldsymbol{F}=E \cdot \Delta \delta
$$


where $\boldsymbol{F}$ is the normal contact force between the blocks, and $\Delta \delta$ is the relative displacement of the contact blocks. In this simulation, the velocity of wave propagation is generated according to the motion of block $\beta_{\mathrm{I}}$, when it is extracted from the pillar. The normal contact force of block $\beta_{\mathrm{I}}$ at each time step is shown in Fig. 35 and the velocity of the wave propagation can be calculated as $V=1819.5 \mathrm{~m} / \mathrm{s}$ following Eqs.(43) and (44). Thus the time of the front blocks separating from the pillar is obtained as $t=1.64 \times 10^{-3} \mathrm{~s}$.

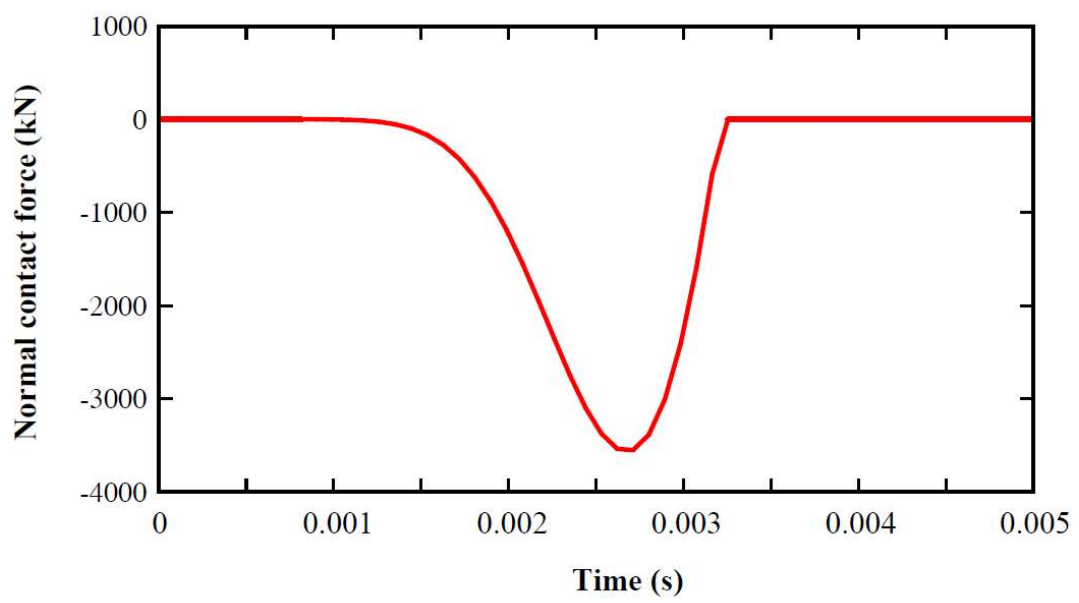

Fig. 35 Time evolution of the normal contact force of block $\beta_{\mathrm{I}}$ for the pillar case.

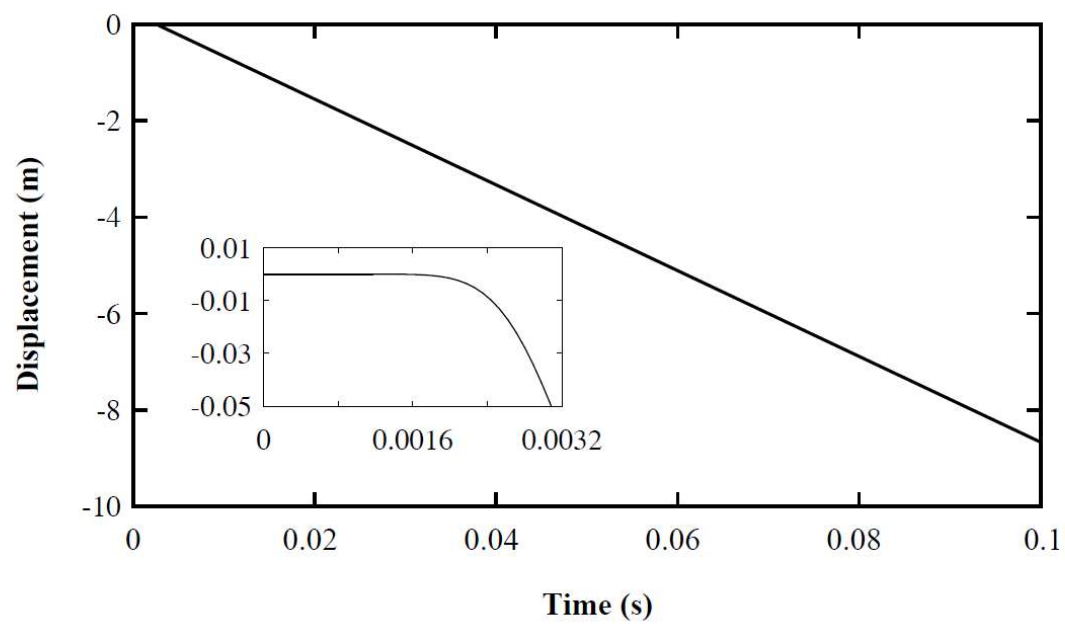

Fig. 36 Time evolution of the displacement in $\mathrm{x}$ axis of block $\beta_{\mathrm{I}}$ for the pillar case.

Time (s)

Fig. 36Fig. 36 exhibits the x-displacement of block A. Significantly, the computationally predicted time of block $\beta_{\mathrm{I}}$ separating from the pillar $t=1.63 \times 10^{-3} \mathrm{~s}$ agrees well the theoretical value $t=1.64 \times 10^{-3} \mathrm{~s}$.

The second kind of wave is the shear wave which propagates transversely in the pillar direction. 
The moving trajectory of the blocks turns into parabolic. The expansion wave in the pillar direction caused by the Poisson effect is ignored in this simulation, because of the rigid physical property of the blocks.

\subsection{Simulation of block accumulation}

A series of numerical simulations of block accumulation are now presented in this example to test the capacity of proposed method in dealing with arbitrary convex polyhedral blocks and illustrate the computational efficiency of the non-uniform block contact detection algorithm.

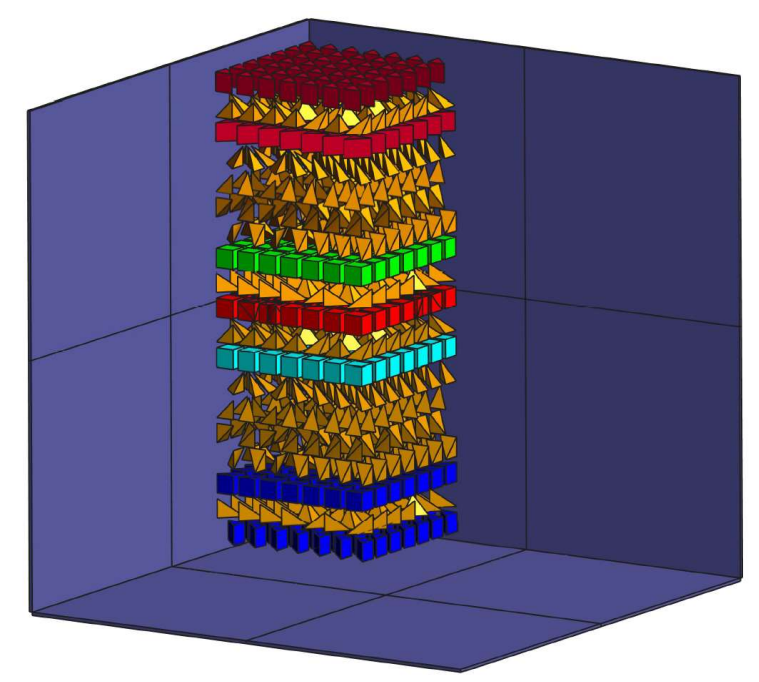

Fig. 37 Deposition of the simulation at initial state.

As shown in Fig. 37, 1093 blocks are accumulated on the ground. The accumulation process consists of two stages. In the first stage, the blocks drop on the ground from a height of $5 \mathrm{~mm}$. In the second stage, these blocks accumulate gradually and finally achieve a stability by applying the same friction coefficient of block-block and block-wall, $\mu=0.4877$ and a damping radio $\xi=0.002$. All the blocks are individual and the geometries are described in Fig. 38. The predicted transient motion of the blocks is presented in Fig. 39.

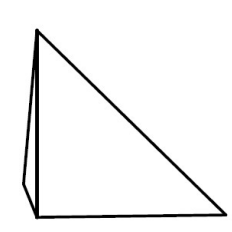

(a)

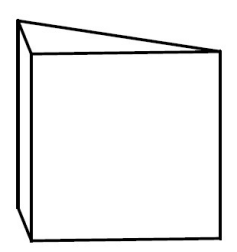

(b)

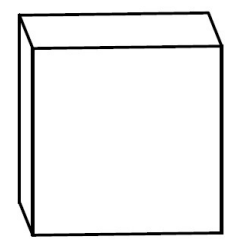

(c)

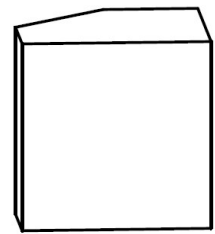

(d)

Fig. 38 The elements utilized in this simulation. 


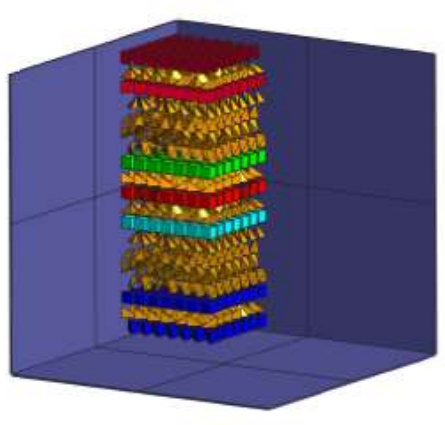

(a)

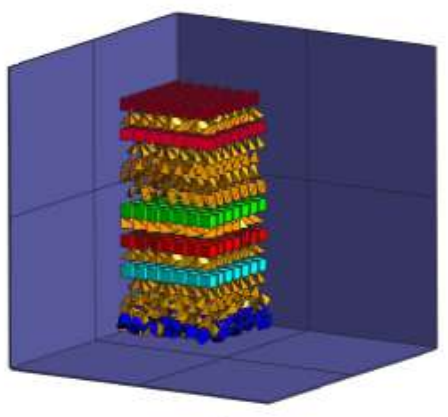

(c)

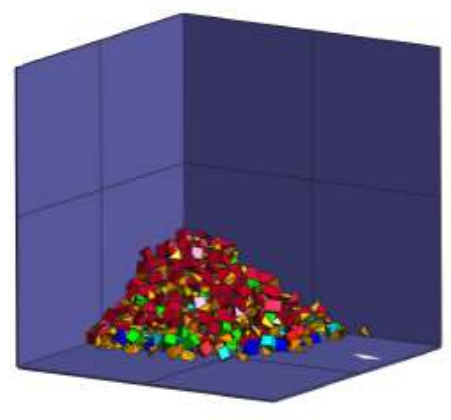

(e)

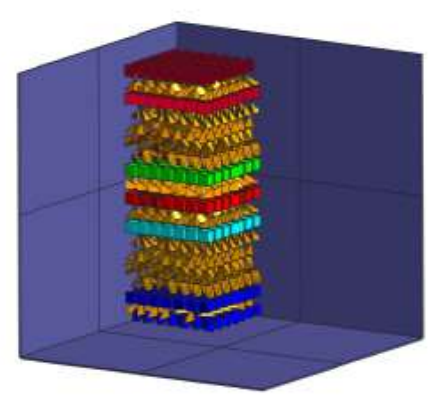

(b)

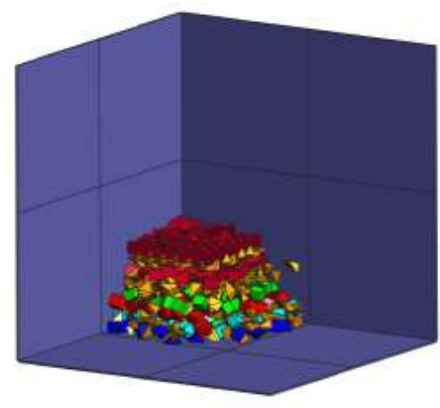

(d)

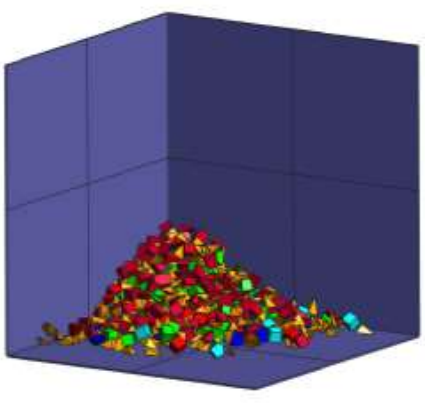

(f)

Fig. 39 The predicted transient motion of the blocks at several times; (a) $t=0 \mathrm{~s}$; (b) $t=0.5 \mathrm{~s}$; (c) $t=1.0$

$$
\mathrm{s} ; \text { (d) } t=2.0 \mathrm{~s} ;(\mathrm{e}) t=3.0 \mathrm{~s} \text {; (f) } t=4.0 \mathrm{~s} \text {. }
$$

The following simulation is used to validate the efficiency of the proposed method to calculate the motion of polyhedral blocks. As shown in Fig. 40(a), a rigid box of dimension $13 \times 13 \times 13 \mathrm{~mm}^{3}$ is adopted and divided as cubes with a length of $1 \mathrm{~mm} .735$ cubes fall into the box and achieve a stable state by applying the same damping ratio of block-block and block-wall, $\xi=0.01$. For comparison, the same example is also simulated with FDEM in which each cube is represented by five tetrahedral blocks bundled together with springs as shown in Fig. 40(b). The simulation methods achieve similar results exhibited in Fig. 41 and both two methods reflect the block motion. However, two approaches present different performance when dealing with this problem of polygonal elements. Table 6 lists the total calculation time of the two methods. It can be seen that it takes nearly quintuple times as long to complete 
this simulaton. Clearly, the proposed method performs much better and it is much more suitable and efficient than the FDEM.

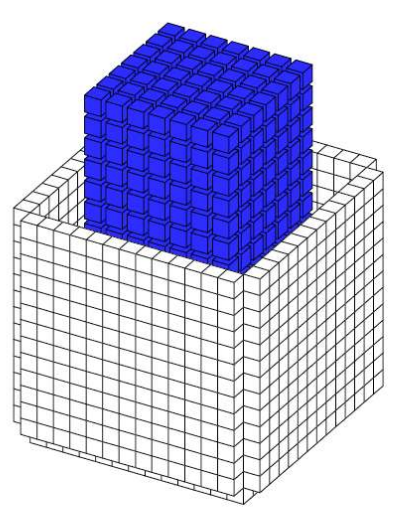

(a)

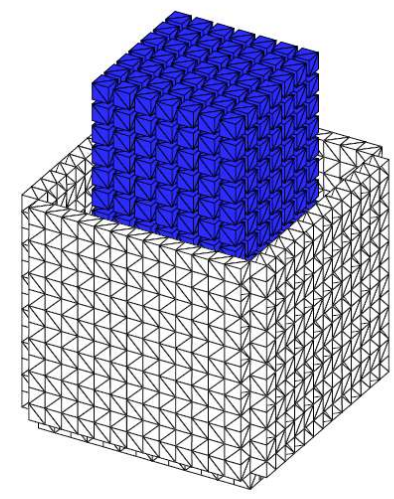

(b)

Fig. 40 Numerical model for this accumulation simulation. (a) the element type of the cube for the proposed method; (b) the element type of five tetrahedral blocks bundled together with springs which have a high elastic stiffness for the FDEM simulation

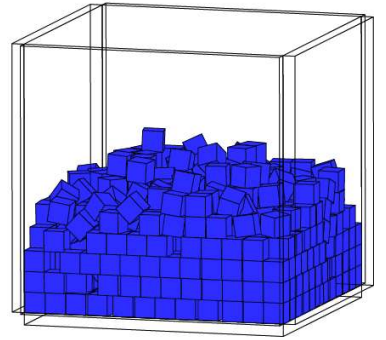

(a)

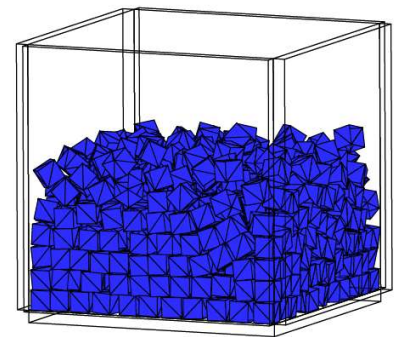

(b)

Fig. 41 Simulation results by the proposed method and FDEM.

Table 6 The computer time required by two methods in simulating the accumulation of blocks with different shapes.

\begin{tabular}{ccc}
\hline & The proposed method & FDEM \\
\hline The CPU time (s) & 16112.14 & 916165.54 \\
\hline
\end{tabular}

The comparison of CPU time taken for the contact detection between the non-uniform blocks contact detection algorithm proposed in this work and NBS contact algorithm is performed in this example using assemblies of 1000, 2000, 3000, 4000, 5000 and 6000 cubic blocks. Two different sizes of cubes used to form the models are described inTable 7, and the distribution is exhibited in Fig. 42. Blocks are dropped on the ground from a height of $2.5 \mathrm{~mm}$. The contact detection is solved ten times for the problem. Each time all contact couples are detected and the CPU time for contact detection is 
measured for the different number of blocks.

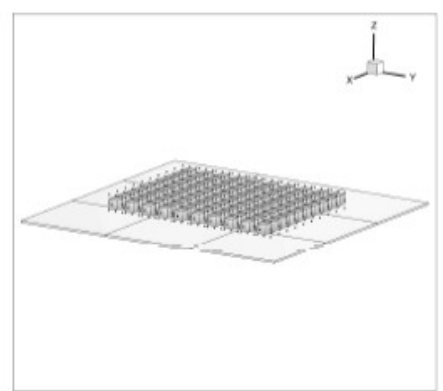

(a)

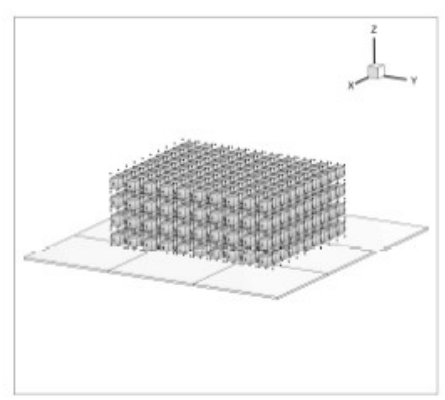

(d)

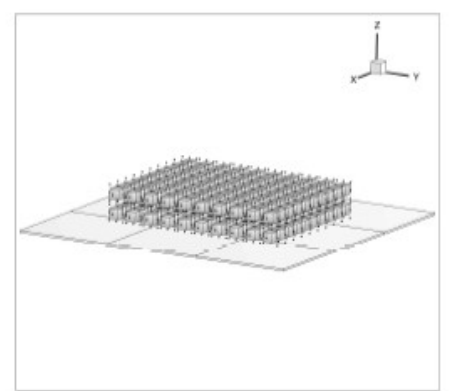

(b)

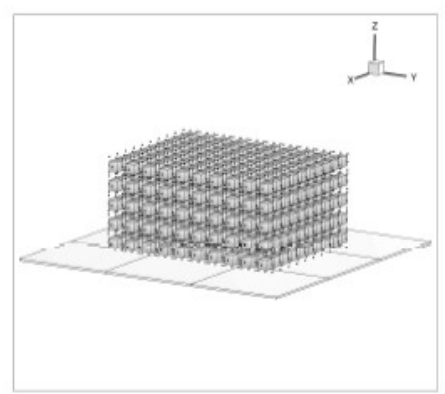

(e)

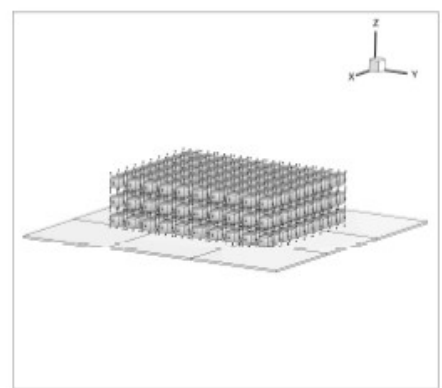

(c)

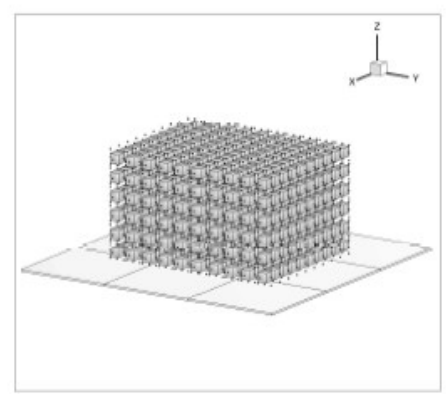

(f)

Fig. 42 Models for the test of efficient test, where the number of blocks is (a) 1000; (b) 2000; (c) 3000; (d) 4000; (e) 5000; (f) 6000 .

Table 7 The geometric properties of the accumulation blocks.

\begin{tabular}{cccc}
\hline Block type & Length $(\mathrm{m})$ & Width $(\mathrm{m})$ & Height $(\mathrm{m})$ \\
\hline Big block & 1.0 & 1.0 & 1.0 \\
\hline Small block & 0.1 & 0.1 & 0.1 \\
\hline
\end{tabular}

The cumulative CPU time by the proposed algorithm is shown in Fig. 43 and it is a linear function of the total number of cubic blocks used in the numerical model. The results are obtained by varying the number of blocks from 1000 to 6000 having the same spatial distribution and density. It is worthy of noticing that the total CPU time is directly proportional to the element number. Fig. 44 shows the simulation results with the NBS contact detection algorithm. The total CPU time is no longer linear and the inefficiency of this method can be observed by comparing with Fig. 43. It is evident that the new contact detection algorithm exhibits a higher computational efficiency in dealing with blocks with different sizes. 


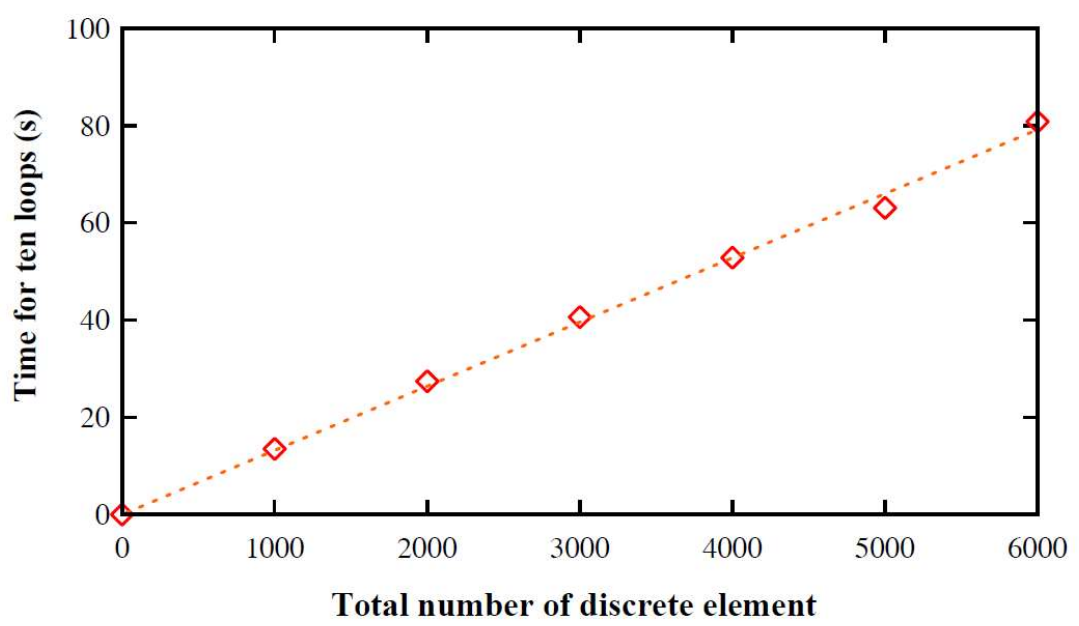

Fig. 43 Total CPU time for ten times contact detections by the proposed contact detection algorithm.

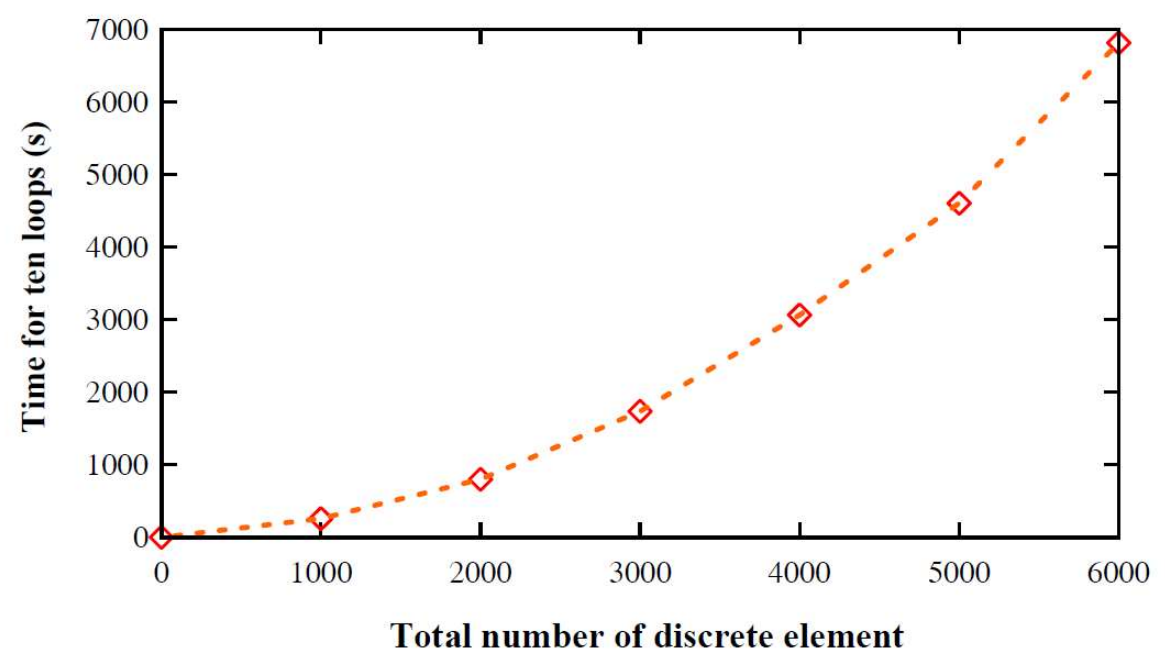

Fig. 44 Total CPU time for ten times contact detections by the NBS contact detection algorithm.

\subsection{Failure process analysis of wedge slope}

Wedge sliding is one of the most common failure forms of the rock slope. In Fig. 45 the analytical model slope with a wedge is shown. The wedge is formed by the surface of slope with left and right structural weak surfaces (LWSP, RWSP). The obliquity of the intersection between the structural surfaces is $\theta$ which is set as 20, 30 and 40 degrees in this simulation. Table 7 shows the physical properties of the slope. In this example, the sliding process of the wedge is simulated under the static and dynamic loading, respectively. 


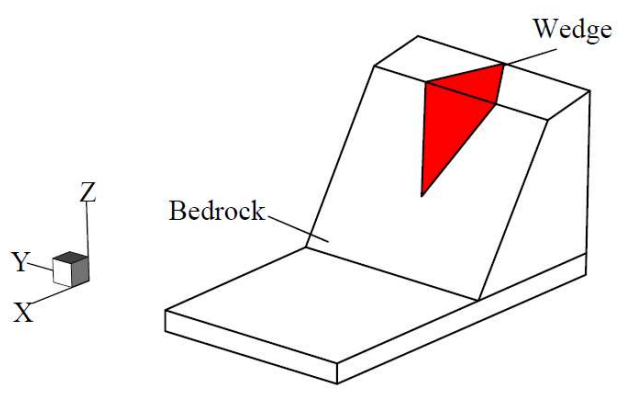

(a)
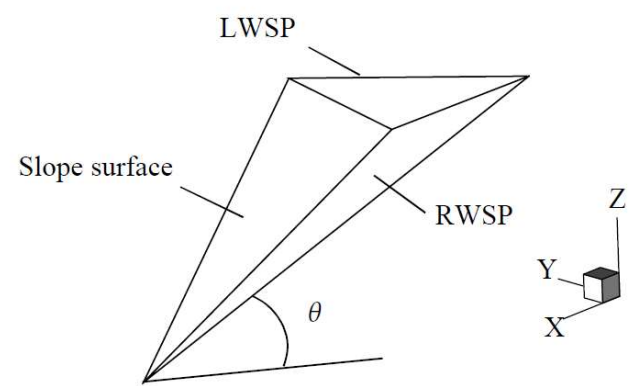

(b)

Fig. 45 Numerical model of the wedge slope, showing (a) the overall model and (b) the geometry of the wedge.

Table 8 Material parameters of the slope.

\begin{tabular}{ccccc}
\hline Density $\left(\mathrm{kg} / \mathrm{m}^{3}\right)$ & $\begin{array}{c}\text { Young's } \\
\text { Modulus(Pa) }\end{array}$ & Poisson ratio & $\begin{array}{c}\text { Normal stiffness } \\
(\mathrm{N} / \mathrm{m})\end{array}$ & $\begin{array}{c}\text { Tangential } \\
\text { stiffness }(\mathrm{N} / \mathrm{m})\end{array}$ \\
\hline 2600 & $2 \times 10^{9}$ & 0.167 & $2 \times 10^{9}$ & $1.2 \times 10^{8}$ \\
\hline
\end{tabular}

In static analysis, the influence of friction coefficient and cohesion on LWSP and RWSR are ignored. The bottom of the slope is fixed on the ground. Thus the wedge starts to slide along the weak structure surfaces driven by the gravity:

$$
s=\frac{1}{2} g t^{2} \cdot \sin \theta
$$

Fig. 46 provides a comparative analysis of the sliding displacement of the wedge between the numerical simulations and the analytical calculations by Eq.(45), revealing excellent match.

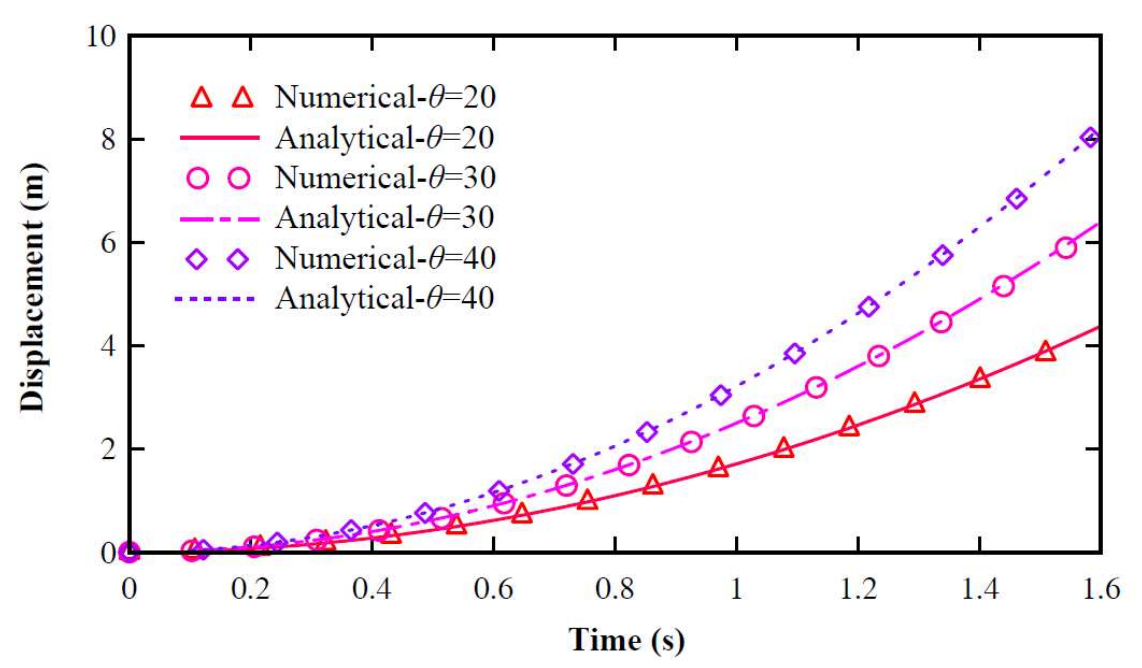

Fig. 46 Time evolution of the displacement of wedge as calculated by the proposed numerical method and the theoretical expression (45). 
The geometry of the wedge for the dynamic analysis is shown in Fig. 47. The numerical model and resisting parameters for this simulation are exhibited in Fig. 48 and Table 9, respectively. Fig. 49 shows the horizontal acceleration-time-history that is applied on the wedge. The velocity and the cumulative displacement variation with time are presented in Fig. 50 and Fig. 51.

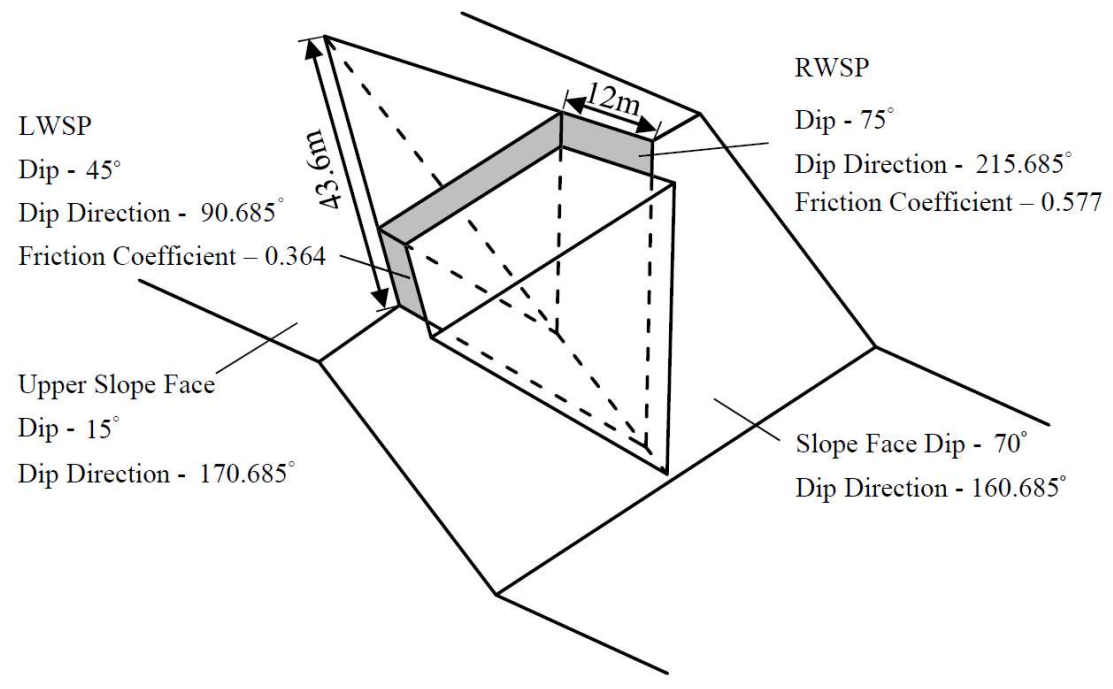

Fig. 47 The wedge considered in this dynamic analysis.

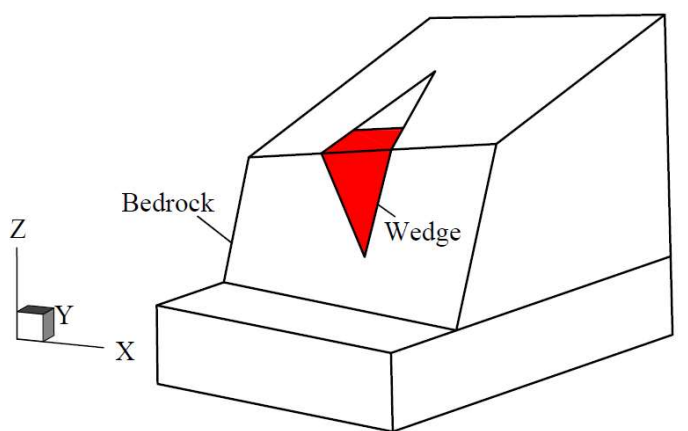

(a)

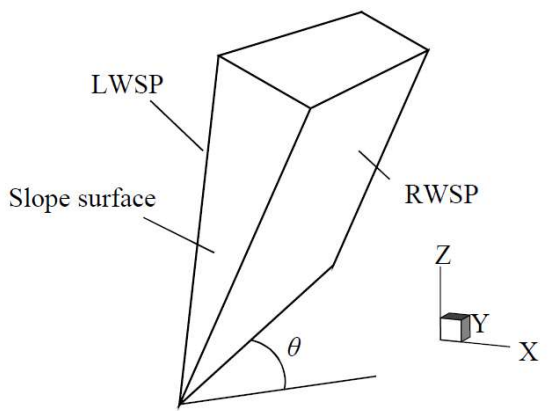

(b)

Fig. 48 Numerical model of the wedge for dynamic analysis, showing (a) the overall model and (b) the geometry of the wedge, $\theta=32.36^{\circ}$.

Table 9 The resisting parameters of the wedge.

\begin{tabular}{ccccc}
\hline $\begin{array}{c}\text { Density } \\
\left(\mathrm{kg} / \mathrm{m}^{3}\right)\end{array}$ & $\begin{array}{c}\text { Young's } \\
\text { Modulus }(\mathrm{Pa})\end{array}$ & Poisson ratio & $\begin{array}{c}\text { Normal stiffness } \\
(\mathrm{N} / \mathrm{m})\end{array}$ & $\begin{array}{c}\text { Tangential } \\
\text { stiffness }(\mathrm{N} / \mathrm{m})\end{array}$ \\
\hline 2000 & $2 \times 10^{9}$ & 0.167 & $2 \times 10^{8}$ & $2 \times 10^{9}$ \\
\hline
\end{tabular}

From the Fig. 50 and Fig. 51 it can be seen that the wedge remains stationary till $t=0.02 \mathrm{~s}$ when the exciting force is greater than the resistance against the sliding. From time $t=0.02 \mathrm{~s}$ to $t=0.629 \mathrm{~s}$ the acceleration is positive. As a result the velocity and displacement continuously increase, while the 
velocity obtains the maximum value at time $t=0.38 \mathrm{~s}$. From $t=0.38 \mathrm{~s}$ to $t=0.629 \mathrm{~s}$, although the acceleration becomes deceleration, the velocity is still greater than zero. Therefore the sliding displacement continues, achieving its maximum value at $t=0.629 \mathrm{~s}$ when the velocity comes to half. At the end of this stage, the displacement has a constant value. This whole phase begins at $t=0.02 \mathrm{~s}$ and lasts until $t=0.8 \mathrm{~s}$. Same motion sequence of the wedge is repeated until the end of the calculation. Excellent agreement is revealed between the computational and analytical predictions for the velocity and displacement in Fig. 50 and Fig. 51, respectively.

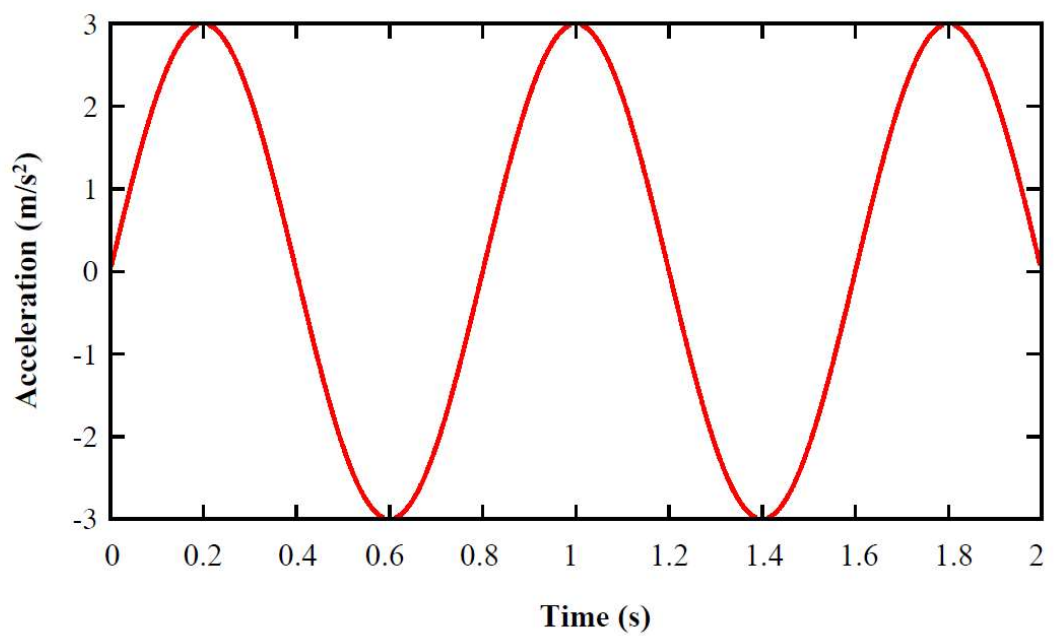

Fig. 49 The horizontal sinusoidal input function.

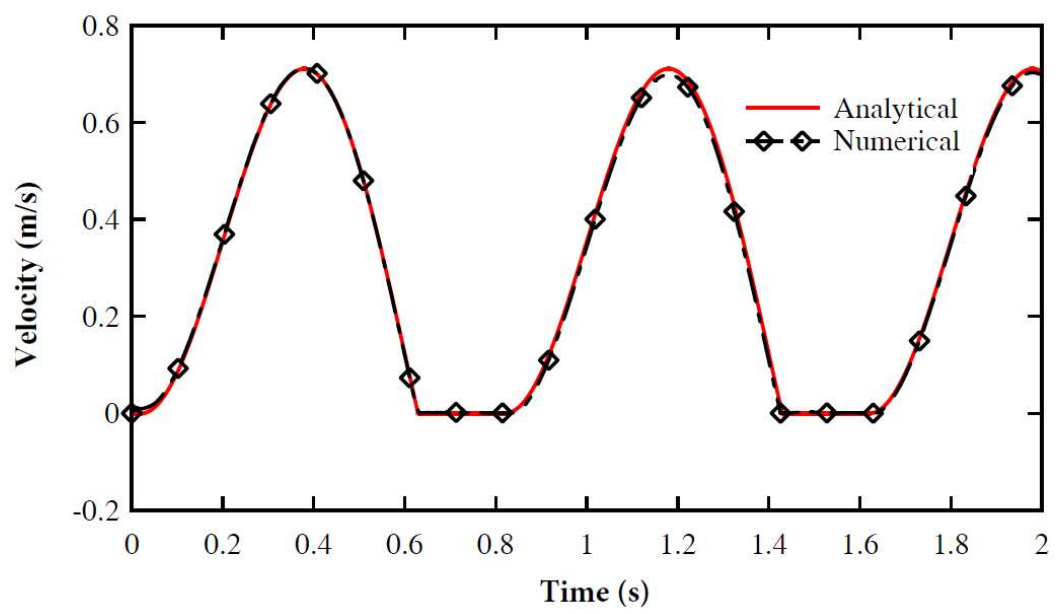

Fig. 50 Time evolution of the velocity of the wedge. 


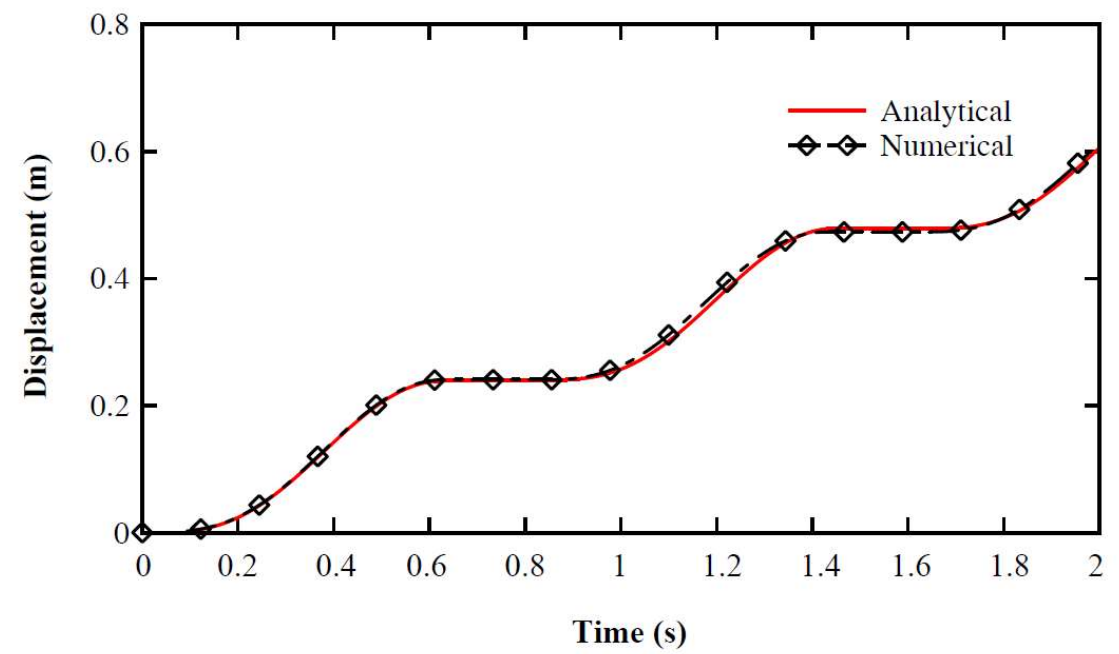

Fig. 51 Time evolution of the displacement of the wedge.

\section{Conclusions}

The current work developed a novel discrete element method using a new definition of a distance potential function. It is applicable for three-dimensional arbitrary convex elements. This newly proposed method has constructed a basic function of the distance potential for an arbitrary convex element, a complete normal contact force calculation model, including the magnitude, direction. The normal contact force moment, is also determined under the concept of the distance potential. A fundamental algorithm is also developed for the tangential contact force. An improved non-uniform block contact detection algorithm for NBS contact detection method is introduced in detail. The calculation method is clear, efficient, and stable for 3D calculations.

To be specific, the main features of the proposed method can be summarized as follows:

(1) Instead of using the standard potential function, the normal contact force is calculated with a new definition of a distance potential function. It presents an accurate potential value and a normal contact force without the influence of the element shape.

(2) Compared with the potential calculation method discussed in FDEM, the proposed method can be implemented to deal with the problems involving arbitrary convex polygonal elements.

(3) The proposed algorithm for the tangential contact force makes the simulation more accurate and reliable.

(4) An improved non-uniform block discrete element search algorithm is introduced to solve the contact detection problem of arbitrary convex elements with different sizes.

\section{Acknowledgement}


This work is supported by the National Natural Science Foundation of China (Grant No. 51279050), the 15th Fok Ying-Tong Education Foundation for Young Teachers in the Higher Education Institutions of China (Grant No. 151073), Non-profit Industry Financial Program of MWR of China (Grant No. 201501034), and the National Key R \& D Program of China (Grant No. 2016YFC0401601).

\section{References}

Shi G, Goodman RE (1985) Two dimensional discontinuous deformation analysis. International Journal for Numerical and Analytical Methods in Geomechanics 9:541-556.

Shi G (2001) Three-dimensional discontinuous deformation analysis. Paper presented at the Proceedings of the Forth International Conference on Analysis of Discontinuous Deformation Glasgow, Scotland, UK, 6-8 June.

Zhang Y, Xu Q, Chen G, Zhao JX, Zheng L (2014) Extension of discontinuous deformation analysis and application in cohesive-frictional slope analysis. International Journal of Rock Mechanics and Mining Sciences 70:533-545.

Nie W, Zhao ZY, Ning Y, Sun JP (2014) Development of rock bolt elements in two-dimensional discontinuous deformation analysis. Rock Mechanics and Rock Engineering 47:2157-2170.

Morgan WE, Aral MM (2015) An implicitly coupled hydro-geomechanical model for hydraulic fracture simulation with the discontinuous deformation analysis. International Journal of Rock Mechanics and Mining Sciences 73:82-94.

Yeung MR, Jiang Q, Sun N (2003) Validation of block theory and three-dimensional discontinuous deformation analysis as wedge stability analysis methods. International Journal of Rock Mechanics and Mining Sciences 40:265-275.

Yeung MR, Jiang Q, Sun N (2007) A model of edge-to-edge contact for three-dimensional discontinuous deformation analysis. Computers and Geotechnics 34:175-186.

Zhu H, Wu W, Chen J, Ma G, Liu X, Zhuang X (2016) Integration of three dimensional discontinuous deformation analysis (DDA) with binocular photogrammetry for stability analysis of tunnels in blocky rockmass. Tunnelling and Underground Space Technology 51:30-40.

Zheng H, Zhang P, Du X (2016) Dual form of discontinuous deformation analysis. Computer Methods in Applied Mechanics and Engineering 305:196-216.

Lin CT, Amadei B, Jung J, Dwyer JF (1996) Extensions of discontinuous deformation analysis for jointed rock masses. International Journal of Rock Mechanics and Mining Sciences and Geomechanics 
Abstracts 33:671-694.

Cai Y, He T, Wang R (2000) Numerical simulation of dynamic process of the tangshan earthquake by a new method-LDDA. Pure and Applied Geophysics 157:2083-2104.

Zheng H, Jiang W (2009) Discontinuous deformation analysis based on complementary theory. Science China-technological Sciences 52:2547-2554.

Li X, Zheng H (2015) Condensed form of complementarity formulation for discontinuous deformation analysis. Science China-technological Sciences 58:1509-1519.

Zheng H, Li X (2015) Mixed linear complementarity formulation of discontinuous deformation analysis. International Journal of Rock Mechanics and Mining Sciences 75:23-32.

Bao H, Zhao Z (2012) The vertex-to-vertex contact analysis in the two-dimensional discontinuous deformation analysis. Advances in Engineering Software 45:1-10.

Shi G (1992) Manifold method of material analysis. Paper presented at the Transaction of the 9th Army conference on applied mathematics and computing, Minneapolis, MN.

Terada K, Asai M, Yamagishi M (2003) Finite cover method for linear and non-linear analyses of heterogeneous solids. International Journal for Numerical Methods in Engineering 58:13211346.

Ning Y, An X, Ma G (2011) Footwall slope stability analysis with the numerical manifold method. International Journal of Rock Mechanics and Mining Sciences 48:964-975.

Zheng H, Xu D (2014) New strategies for some issues of numerical manifold method in simulation of crack propagation. International Journal for Numerical Methods in Engineering 97:986-1010.

Jiang Q, Zhou C, Li D (2009) A three-dimensional numerical manifold method based on tetrahedral meshes. Computers and Structures 87:880-889.

He L, An X, Ma G, Zhao Z (2013) Development of three-dimensional numerical manifold method for jointed rock slope stability analysis. International Journal of Rock Mechanics and Mining Sciences 64:22-35.

Jing L (2003) A review of techniques, advances and outstanding issues in numerical modelling for rock mechanics and rock engineering. International Journal of Rock Mechanics and Mining Sciences 40:283-353.

Cundall PA, Strack OD (1979) A discrete numerical model for granular assemblies. Geotechnique 29:4765. 
Smeets B, Odenthal T, Vanmaercke S, Ramon H (2015) Polygon-based contact description for modeling arbitrary polyhedra in the discrete element method. Computer Methods in Applied Mechanics and Engineering 290:277-289.

Hohner D, Wirtz S, Kruggelemden H, Scherer V (2011) Comparison of the multi-sphere and polyhedral approach to simulate non-spherical particles within the discrete element method: Influence on temporal force evolution for multiple contacts. Powder Technology 208:643-656.

Itasca (2014a) PFC. 2D (Particle flow code in 2 dimensions), 5.0 edn. Itasca Consulting Group, Minneapolis.

Itasca (2014b) PFC. 3D (Particle flow code in 3 dimensions), 5.0 edn. Itasca Consulting Group, Minneapolis.

Albusaidi A, Hazzard JF, Young RP (2005) Distinct element modeling of hydraulically fractured Lac du Bonnet granite. Journal of Geophysical Research 110:B06032.

Shimizu H, Murata S, Ishida T (2011) The distinct element analysis for hydraulic fracturing in hard rock considering fluid viscosity and particle size distribution. International Journal of Rock Mechanics and Mining Sciences 48:712-727.

Yoon JS, Zang A, Stephansson O (2014) Numerical investigation on optimized stimulation of intact and naturally fractured deep geothermal reservoirs using hydro-mechanical coupled discrete particles joints model. Geothermics 52:165-184.

Mcdowell GR, Harireche O (2002) Discrete element modelling of soil particle fracture. Geotechnique 52:131-135.

Jung JW, Santamarina JC, Soga K (2012) Stress-strain response of hydrate-bearing sands: Numerical study using discrete element method simulations. Journal of Geophysical Research 117:B04202.

Wang J, Yan H (2013) On the role of particle breakage in the shear failure behavior of granular soils by DEM. International Journal for Numerical and Analytical Methods in Geomechanics 37:832854.

Garcia X, Latham J, Xiang J, Harrison JP (2009) A clustered overlapping sphere algorithm to represent real particles in discrete element modelling. Geotechnique 59:779-784.

Zhou B, Huang R, Wang H, Wang J (2013) DEM investigation of particle anti-rotation effects on the micromechanical response of granular materials. Granular Matter 15:315-326.

Latham J, Munjiza A (2004) The modelling of particle systems with real shapes. Philosophical 
Transactions of the Royal Society A 362:1953-1972.

Jiang M, Shen Z, Wang J (2015) A novel three-dimensional contact model for granulates incorporating rolling and twisting resistances. Computers and Geotechnics 65:147-163.

Cundall PA (1971) A computer model for simulating progressive, large-scale movements in blocky rock systems. Paper presented at the Proceedings of the International Symposium on Rock Fracture, Nancy.

Cundall PA (1988) Formulation of a three-dimensional distinct element model-Part I. A scheme to detect and represent contacts in a system composed of many polyhedral blocks. International Journal of Rock Mechanics and Mining Sciences and Geomechanics Abstracts 25:107-116.

Hart R, Cundall PA, Lemos J (1988) Formulation of a three-dimensional distinct element model—Part II. Mechanical calculations for motion and interaction of a system composed of many polyhedral blocks. International Journal of Rock Mechanics and Mining Sciences and Geomechanics Abstracts 25:117-125.

Cundall PA, Hart RD (1985) Development of Generalized 2-D and 3-D distinct element programs for modeling jointed rock. Itasca Consulting Group. Misc. Paper SL-85-1. U.S. Army Corps of Engineering.

Itasca (2016a) UDEC-universal distinct element code, 6.0 edn. Itasca Consulting Group, Minneapolis.

Itasca (2016b) 3DEC-3-D Distinct Element Code, 6.0 edn. Itasca Consulting Group, Minneapolis.

Zhu JB, Deng X, Zhao XB, Zhao J (2013) A numerical study on wave transmission across multiple intersecting joint sets in rock masses with UDEC. Rock Mechanics and Rock Engineering 46:1429-1442.

Boon CW, Houlsby GT, Utili S (2014) New insights into the 1963 Vajont slide using 2D and 3D distinctelement method analyses. Geotechnique 64:800-816.

Nezami EG, Hashash YMA, Zhao D, Ghaboussi J (2004) A fast contact detection algorithm for 3-D discrete element method. Computers and Geotechnics 31:575-587.

Nezami EG, Hashash YMA, Zhao D, Ghaboussi J (2006) Shortest link method for contact detection in discrete element method. International Journal for Numerical and Analytical Methods in Geomechanics 30:783-801.

Li S, Zhao M, Wang Y, Rao Y (2004) A new numerical method for dem - block and particle model. International Journal of Rock Mechanics and Mining Sciences 41:414-418. 
Jin F, Zhang C, Hu W, Wang J (2011) 3D mode discrete element method: Elastic model. International Journal of Rock Mechanics and Mining Sciences 48:59-66.

Feng YT, Han K, Owen DRJ (2012) Energy-conserving contact interaction models for arbitrarily shaped discrete elements. Computer Methods in Applied Mechanics and Engineering 205:169-177.

Lu G, Third JR, Muller CR (2015) Discrete element models for non-spherical particle systems: From theoretical developments to applications. Chemical Engineering Science 127:425-465.

Kawamoto R, Ando E, Viggiani G, Andrade JE (2016) Level set discrete element method for threedimensional computations with triaxial case study. Journal of The Mechanics and Physics of Solids 91:1-13.

Kodam M, Bharadwaj R, Curtis JS, Hancock BC, Wassgren C (2010a) Cylindrical object contact detection for use in discrete element method simulations. Part I - Contact detection algorithms. Chemical Engineering Science 65:5852-5862.

Kodam M, Bharadwaj R, Curtis JS, Hancock BC, Wassgren C (2010b) Cylindrical object contact detection for use in discrete element method simulations, Part II-Experimental validation. Chemical Engineering Science 65:5863-5871.

Munjiza A, Owen DRJ, Bicanic N (1995) A combined finite-discrete element method in transient dynamics of fracturing solids. Engineering Computations 12:145-174.

Munjiza A, Andrews KRF (1998) NBS contact detection algorithm for bodies of similar size. International Journal for Numerical Methods in Engineering 43:131-149.

Munjiza A, Rougier E, John NWM (2006) MR linear contact detection algorithm. International Journal for Numerical Methods in Engineering 66:46-71.

Munjiza A (2004) The Combined Finite-Discrete Element Method. Wiley, Chichester, West Sussex, Hoboken.

Mahabadi OK, Grasselli G, Munjiza A (2010) Y-GUI: A graphical user interface and pre-processor for the combined finite-discrete element code, $\mathrm{Y} 2 \mathrm{D}$, incorporating material heterogeneity. Computers and Geosciences 36:241-252.

Munjiza A, John NWM (2002) Mesh size sensitivity of the combined FEM/DEM fracture and fragmentation algorithms. Engineering Fracture Mechanics 69:281-295.

Munjiza A, Bangash T, John NWM (2004) The combined finite-discrete element method for structural failure and collapse. Engineering Fracture Mechanics 71:469-483. 
Rougier E, Bradley CR, Broom ST, Knight EE, Munjiza A, Sussman AJ, Swift RP (2011) The Combined Finite-Discrete Element Method applied to the Study of Rock Fracturing Behavior in 3D. In: American Rock Mechanics Association 45 th US Rock Mechanics/Geomechanics Symposium, San Francisco, USA, 26-29 June.

Mahabadi OK, Lisjak A, Munjiza A, Grasselli G (2012) Y-Geo: New combined finite-discrete element numerical code for geomechanical applications. International Journal of Geomechanics 12:676688.

Yan CZ, Zheng H, Ge XR (2015) Unified calibration based potential contact force in discrete element method. Rock and Soil Mechanics 36:249-256.

Yan CZ, Zheng H (2016) A new potential function for the calculation of contact forces in the combined finite-discrete element method. International Journal for Numerical and Analytical Methods in Geomechanics 41:265-28.

Li SH, Wang J, Liu B, Dong DP (2007) Analysis of critical excavation depth for a jointed rock slope using a face-to-face discrete element method. Rock Mechanics and Rock Engineering 40:331348. 